\title{
Inositol-triphosphate 3-kinase B confers cisplatin resistance by regulating NOX4-dependent redox balance
}

\author{
Chaoyun Pan, ${ }^{1}$ Lingtao Jin, ${ }^{1}$ Xu Wang, ${ }^{1}$ Yuancheng Li, ${ }^{2}$ Jaemoo Chun, ${ }^{1}$ Austin C. Boese, ${ }^{1}$ Dan Li, ${ }^{1}$ Hee-Bum Kang, ${ }^{1}$ Guojing Zhang, \\ Lu Zhou, ${ }^{3}$ Ceorgia Z. Chen, ${ }^{1}$ Nabil F. Saba, ${ }^{1}$ Dong M. Shin, ${ }^{1}$ Kelly R. Magliocca, ${ }^{4}$ Taofeek K. Owonikoko, ${ }^{1}$ Hui Mao, ${ }^{2}$ \\ Sagar Lonial, ${ }^{1}$ and Sumin Kang ${ }^{1}$ \\ 'Winship Cancer Institute, Department of Hematology and Medical Oncology, and ²Department of Radiology and Imaging Sciences, Emory University School of Medicine, Atlanta, Ceorgia, USA. ${ }^{3}$ Department of \\ Chemistry and Institute for Biophysical Dynamics, The University of Chicago, Chicago, Illinois, USA. ${ }^{4}$ Department of Pathology and Laboratory Medicine, Emory University School of Medicine, Atlanta, Georgia, USA.
}

\begin{abstract}
How altered metabolism contributes to chemotherapy resistance in cancer cells remains unclear. Through a metabolismrelated kinome RNAi screen, we identified inositol-trisphosphate 3-kinase B (ITPKB) as a critical enzyme that contributes to cisplatin-resistant tumor growth. We demonstrated that inositol 1,3,4,5-tetrakisphosphate (IP4), the product of ITPKB plays a critical role in redox homeostasis upon cisplatin exposure by reducing cisplatin-induced ROS through inhibition of a ROS-generating enzyme, NADPH oxidase 4 (NOX4), which promotes cisplatin-resistant tumor growth. Mechanistically, we identified that IP4 competes with the NOX4 cofactor NADPH for binding and consequently inhibits NOX4. Targeting ITPKB with shRNA or its small-molecule inhibitor resulted in attenuation of NOX4 activity, imbalanced redox status, and sensitized cancer cells to cisplatin treatment in patient-derived xenografts. Our findings provide insight into the crosstalk between kinase-mediated metabolic regulation and platinum-based chemotherapy resistance in human cancers. Our study also suggests a distinctive signaling function of IP4 that regulates NOX4. Furthermore, pharmaceutical inhibition of ITPKB displayed synergistic attenuation of tumor growth with cisplatin, suggesting ITPKB as a promising synthetic lethal target for cancer therapeutic intervention to overcome cisplatin resistance.
\end{abstract}

\section{Introduction}

Cisplatin kills rapidly dividing cells by damaging their DNA and plays a crucial role in the chemotherapy of human cancers (1-3). Nevertheless, cisplatin is often accompanied by side effects, and the development of resistance limits its curative potential. Patients with chemotherapy-resistant and recurrent cancers often have an extremely poor prognosis. Therefore, understanding critical factors determining cisplatin resistance is needed to improve the therapeutic outcomes of cisplatin-based chemotherapy.

Emerging evidence supports that dysregulated cellular metabolism is associated with resistance to cancer chemotherapy $(4,5)$. Studies have demonstrated that the metabolic landscape in tumor cells that are resistant to cisplatin differs from that in cisplatin-sensitive cells. For instance, cisplatin-resistant ovarian or cervical cancer cells undergo a metabolic shift to rely more on oxidative metabolism and become less addicted to the glycolytic pathway $(6,7)$. In addition, reprogrammed lipid metabolism is observed in cisplatin-resistant bladder cancer cells (8). Increased reactive oxygen species (ROS) and an altered redox status are observed in cancer cells compared with normal proliferating cells $(9,10)$. Chemotherapy, including cisplatin, often results in further elevated ROS, and the overproduced ROS leads

Conflict of interest: The authors have declared that no conflict of interest exists. Copyright: ( 2019, American Society for Clinical Investigation.

Submitted: August 28, 2018; Accepted: March 26, 2019; Published: May 13, 2019. Reference information: J Clin Invest. 2019;129(6):2431-2445.

https://doi.org/10.1172/JCl124550. to cancer cell death once it exceeds a toxic threshold. Cancer cells possess a series of ROS-regulating enzymes to manage the ROS balance (11). Thus, dysregulation of these enzymes may lead to imbalanced redox homeostasis and consequent chemotherapyresistant tumor growth.

Several signaling factors have been identified that provide a potential link between cancer metabolism and cancer cisplatin resistance. HIF1 confers cisplatin resistance by regulating metabolic enzymes involved in the glycolysis pathway in ovarian cancer (12). Targeting breast cancer gene 2 (BRCA2) excites the cisplatin response and modulates cellular respiration in lung cancer cells (13). Sirtuin 1 (SIRT1), a histone deacetylase, contributes to cell viability in cisplatin-resistant cervical cancer cells in which mitochondrial metabolism is altered (14). Comparative lipidomic profiling demonstrated that acetyl-CoA synthesis 2 (ACSS2) is upregulated in cisplatin-resistant bladder cancer cells (8). Antioxidant thioredoxin 1 (TRX1) of the redox regulation system is known to impact ROS and oxidative metabolism, contributing to cisplatin resistance in lung cancer (15), and cisplatin induces ROS production by the activation of NADPH oxidase (NOX) in human prostate cancer cells (16). The expression level of NADPH oxidase 4 (NOX4) is increased in tumors compared with adjacent tissues in glioblastoma patients (17), and cisplatin induces NOX4 expression in murine cells and causes acute kidney injury through ROS-mediated programmed cell death $(18,19)$. Although these studies have identified signaling factors that are involved in metabolism and contribute to the cisplatin response in human cancers, most 
A

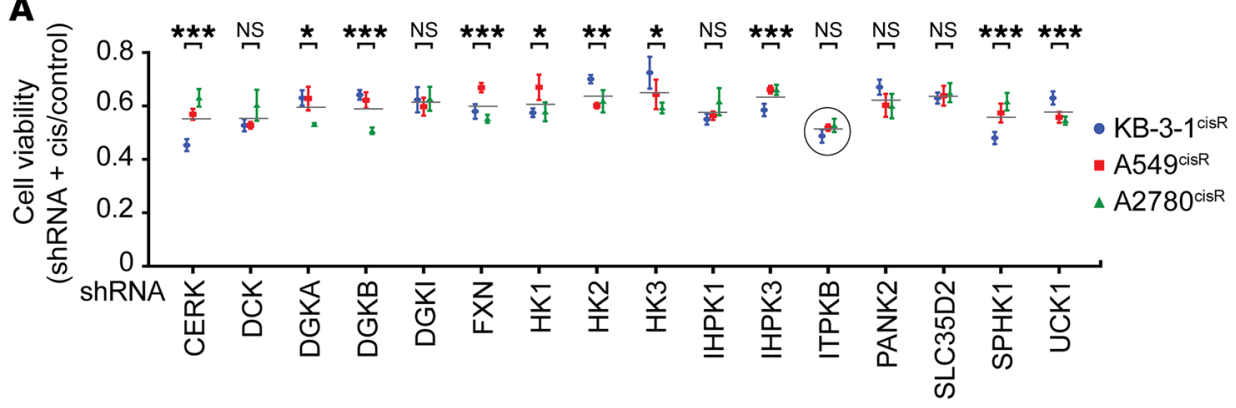

B

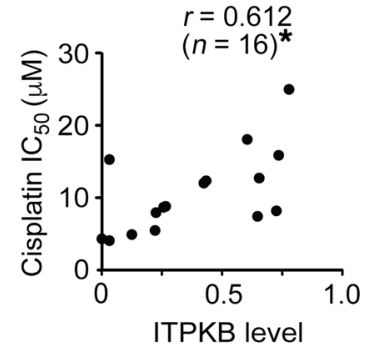

Cell lines: HNSCC

C

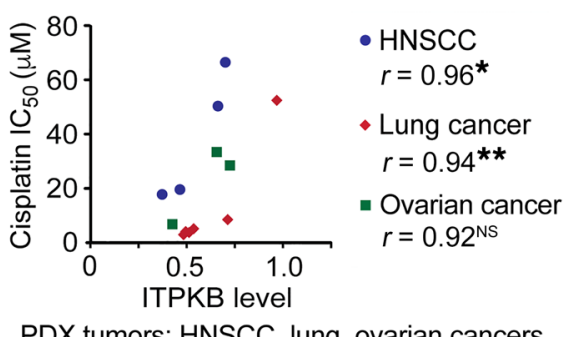

PDX tumors: HNSCC, lung, ovarian cancers

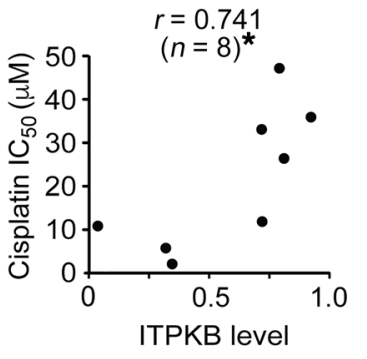

Lung cancer

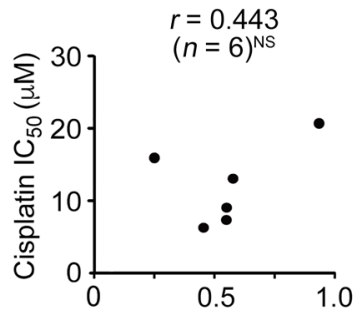

ITPKB level

Ovarian cancer

to mediate the survival signaling of $\mathrm{B}$ cells and $\mathrm{T}$ cell receptor signaling (26-29). In addition, ITPKB controls hematopoietic stem cell homeostasis through AKT and mTOR signaling (30). However, few studies link ITPKB to human solid tumors. ITPKA is implicated in actin remodeling, which contributes to migratory and metastatic potential in breast cancer and lung cancer (31). Here, we report that the kinase ITPKB provides a metabolic advantage and confers cisplatin resistance by controlling redox homeostasis in human cancers.

\section{Results}

ITPKB expression positively correlates with cisplatin resistance in diverse human cancers. To better understand the link between protein kinase signaling in metabolic regulation and its contribution to cancer cisplatin resistance, we used a human kinome-wide lentiviral shRNA library to identify metabolic drivers that may impact cisplatin resistance. Two-step screening was performed to first select genes from 781 kinase and kinase-related genes that are only lethal when target-downregulated in the presence of a sublethal dose of cisplatin. Among the top 100 ranking genes in the primary screen, 16 kinases using metabolites as substrates were selected. These metabolism-related genes were evaluated in 3 different cisplatin-resistant (cisplatinresistant) cancer cell lines - lung cancer A549 ${ }^{\text {cisR }}$, ovarian cancer A2780 ${ }^{\text {cisR }}$, and cervical cancer KB3-1 ${ }^{\text {cisR }}$ cells (Supplemental Figure 1A; supplemental material available online with this article; https://doi.

studies focus on observations or correlations, which poses limits to deciphering the mechanistic bases.

In this study, through a metabolism-related kinome RNAi screen, we identified inositol-triphosphate 3-kinase B (ITPKB) as a central driver of cisplatin resistance in human cancers. ITPK metabolizes inositol 1,4,5-trisphosphate (IP3) to inositol 1,3,4,5-tetrakisphosphate (IP4) by catalyzing the $\gamma$-phosphate from ATP to the $3^{\prime}$ position of IP3 $(20,21)$. ITPK isoforms ITPKA, ITPKB, and ITP$\mathrm{KC}$ are highly conserved in their catalytic active domain (22). The small-molecule inhibitors BAMB-4 and GNF362 are reported to specifically inhibit ITPK isoforms (23-25). Mounting evidence suggests that ITPKB is implicated in hematopoiesis. ITPKB is known
org/10.1172/JCI124550DS1) - the cisplatin-resistant features of which are described in Methods. ITPKB, which converts IP3 to IP4, was identified as the most effective common target from the secondary screen by sensitizing cancer cells to cisplatin treatment across 3 cancer types (Figure 1A).

To explore the relationship between ITPKB expression and cisplatin resistance in human cancers, we examined ITPKB expression and cisplatin sensitivity in 22 human cancer cell lines and 13 patient-derived xenograft (PDX) tumors of head and neck squamous cell carcinoma (HNSCC), lung cancer, and ovarian cancer. ITPKB level and cisplatin resistance positively correlated in both cancer cell lines and PDX tumors (Figure 1, B and C, and Supplemental Figure 
A
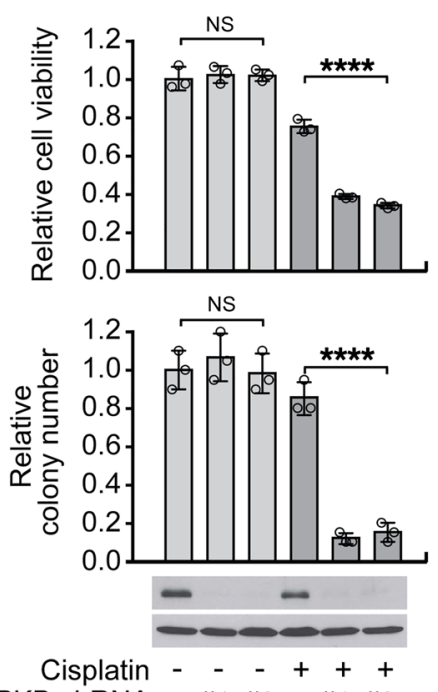

ITPKB shRNA - \#1 \#2 - \#1 \#2

KB-3-1 ${ }^{\text {cisR }}$
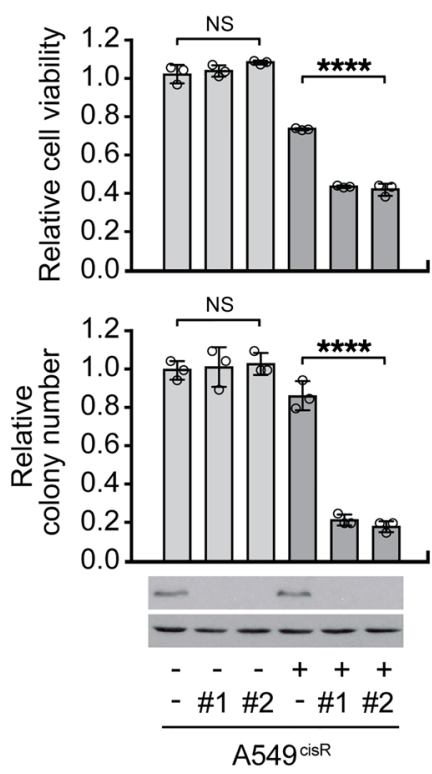
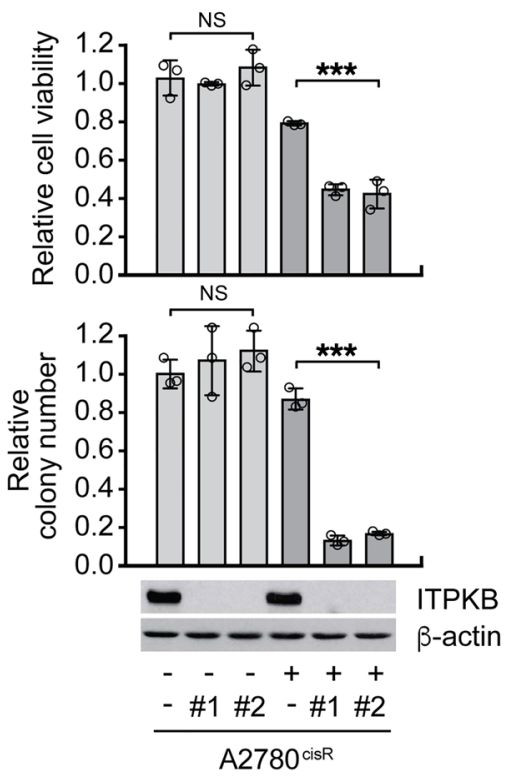

B

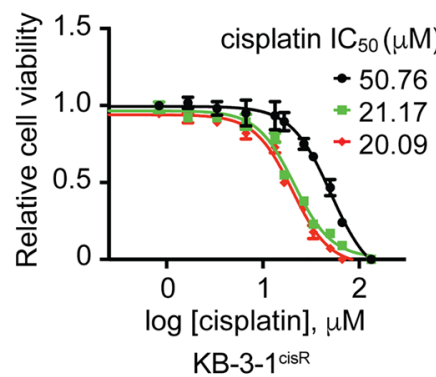

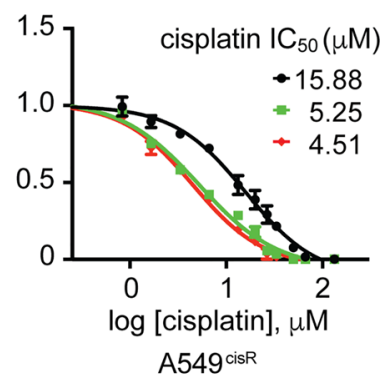
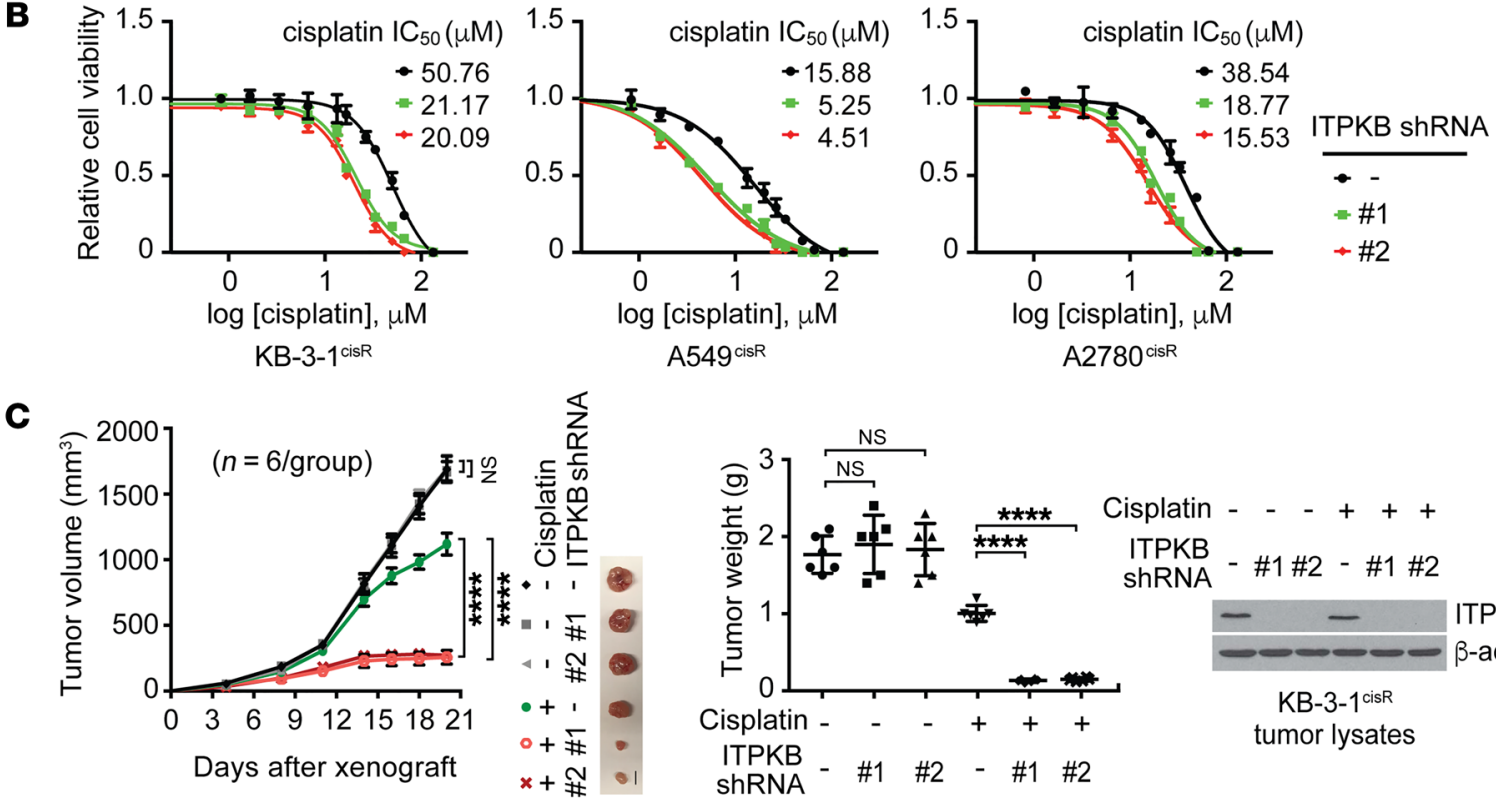

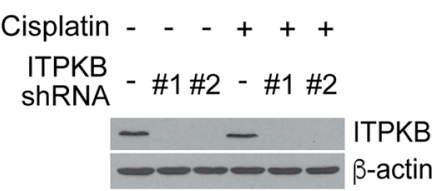

KB-3-1 ${ }^{\text {cisR }}$

tumor lysates

Figure 2. ITPKB is important for cisplatin-resistant cancer cell proliferation and tumor growth. (A) Cell viability (top) and colony formation potential (bottom) of KB-3-7 cisR $^{\text {is }}$ A49 ${ }^{\text {cisR }}$, and A2780 cisR cells with ITPKB knockdown. Cells were transduced with ITPKB shRNA clones and treated with sublethal doses of cisplatin (KB-3-1 $1^{\text {cisR }}, 5 \mu \mathrm{g} / \mathrm{ml}$; A549 ${ }^{\text {cisR }}, 2 \mu \mathrm{g} / \mathrm{ml}$; A2780 ${ }^{\text {cisR }}, 5 \mu \mathrm{g} / \mathrm{ml}$ ). Knockdown efficiency of ITPKB is shown by immunoblotting. (B) Cisplatin IC ${ }_{50}$ in KB-3-7 cisR $^{\text {, }}$ $A 549^{\text {cisR }}$, and A2780 ${ }^{\text {cisR }}$ cells with ITPKB knockdown. Cells were treated with increasing concentrations of cisplatin for 48 hours. (C) Effect of ITPKB knockdown and cisplatin treatment on tumor growth. Mice were treated with PBS or cisplatin ( $5 \mathrm{mg} / \mathrm{kg}$ i.p. twice per week) from 3 days after xenograft, and tumor size (left) and tumor weight (middle) were monitored. Knockdown of ITPKB in tumors is shown by immunoblotting (right). Scale bars represent $10 \mathrm{~mm}$ for tumor size. (A and B) Data are mean \pm SD from 3 technical replicates of each sample and are representative of 4 (A) and 2 (B) independent biological experiments. (C) Error bars represent SEM for tumor volume and SD for tumor weight $(n=6)$. Statistical analysis was performed by 2 -way ANOVA for tumor volume $(C$, left) and 1-way ANOVA for all other data $\left({ }^{* * *} P<0.005 ;{ }^{* * *} P<0.0001\right)$.

$1, \mathrm{~B}$ and $\mathrm{C})$. Interestingly, PDX tumors, which are more clinically relevant than cell lines, demonstrated stronger positive correlation with $r$ value of 0.92-0.96 compared with cancer cell lines $(r=0.44-$ 0.74). Furthermore, ITPKB expression and its relationship to cisplatin resistance were further investigated in primary patient tumor specimens. Primary tumors from HNSCC patients who received platinum-based chemotherapy including cisplatin and carboplatin were stained for ITPKB (Supplemental Figure 1D). HNSCC patients were separated into 2 groups: patients who responded to platinum therapy for a duration of 2 years and patients who lost response within the 2-year period and had regrowth of tumors off treatment. The group of tumors from patients who had recurrent disease within 2 years ("no response" group) had higher expression of ITPKB compared with the group who responded to platinum therapy for over 2 years ("response" group) (Figure 1D and Supplemental Figure 1E). Clinical information for all patients from whom primary HNSCC 
A

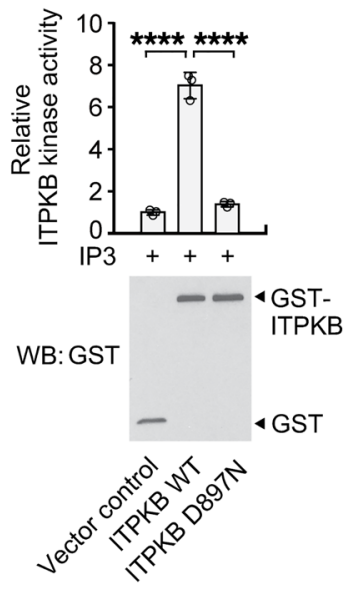

(D897N: DN)
B

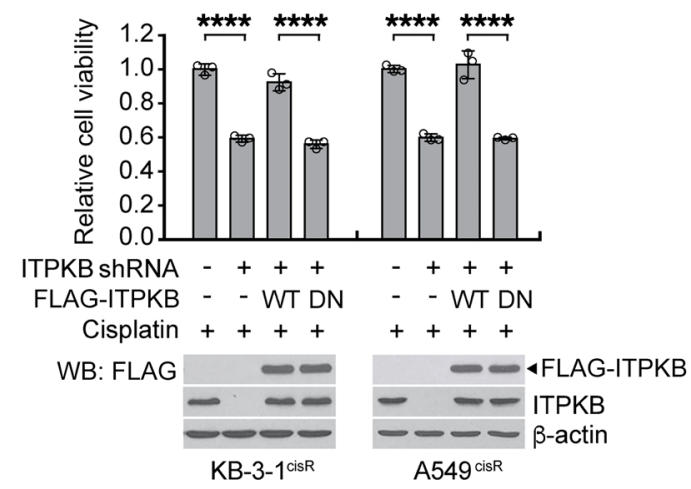

D

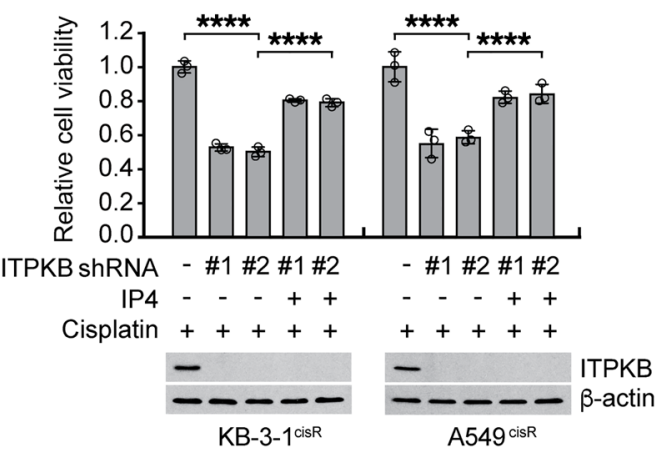

C

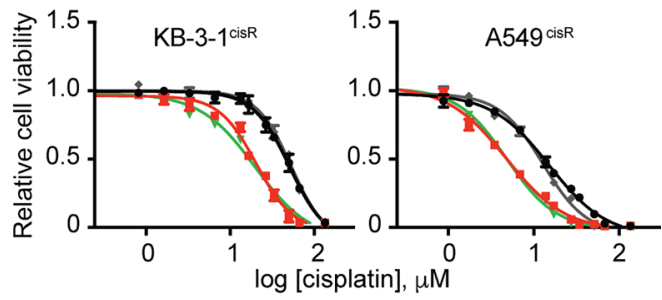

cisplatin $\mathrm{IC}_{50}(\mu \mathrm{M})$

- pLKO1

$50.94 \quad 14.69$

20.50

4.61

- ITPKB shRNA + FLAG-ITPKB WT $53.40 \quad 12.43$

- ITPKB shRNA + FLAG-ITPKB DN $\frac{19.96}{\mathrm{~KB}-3-1^{\text {cisR }}} \frac{4.63}{\mathrm{~A} 549^{\text {cisR }}}$

E

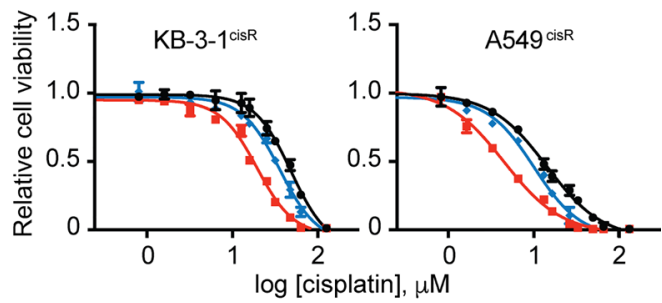

- pLKO1

$\frac{\text { cisplatin } \mathrm{IC}_{50}(\mu \mathrm{M})}{51.18 \quad 14.37}$

- ITPKB shRNA

$20.34 \quad 4.54$

- ITPKB shRNA + IP4

$\frac{37.81}{K_{B}-3-1^{\text {cisR }}} \frac{10.24}{A 549^{\text {cisR }}}$
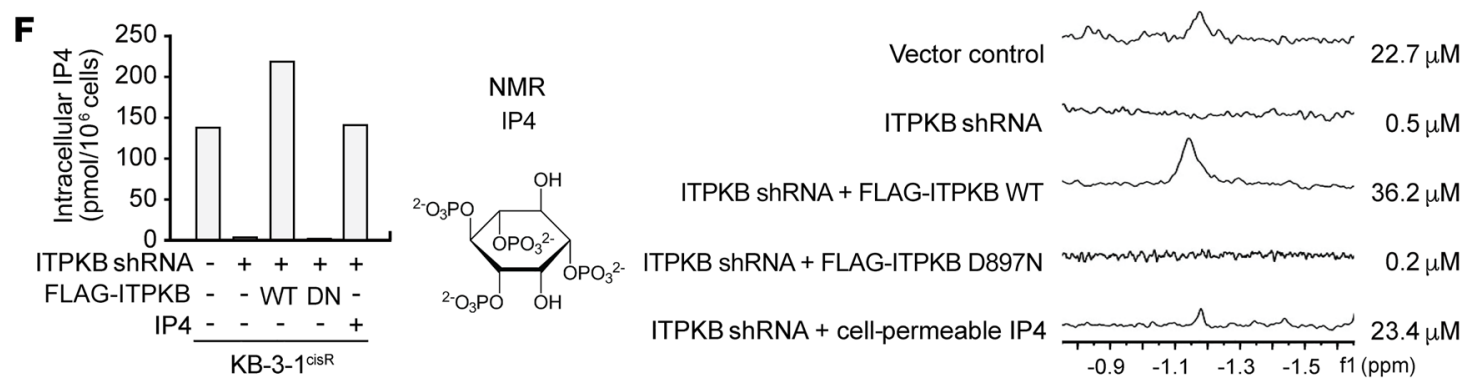

Figure 3. IP4, the product of ITPKB, confers cisplatin resistance to cancer cells. (A) In vitro ITPKB kinase assay using IP3 as a substrate. ITPKB WT and kinase-dead mutant D897N were enriched from 293T by GST pulldown assay. Kinase activity of ITPKB was assessed using ADP-Clo kinase assay. (B and C) Effect of ITPKB WT or D897N rescue expression and ITPKB knockdown on cell viability (B) and cisplatin sensitivity (C). Cells with ITPKB variants were treated with a sublethal dose of cisplatin (KB-3-1 cisR $^{\text {is }} 5 \mu \mathrm{g} / \mathrm{ml}$; A549 ${ }^{\text {cisR }, ~} 2 \mu \mathrm{g} / \mathrm{ml}$ ) for 48 hours, and viability was measured using CellTiter-Clo assay. Cisplatin sensitivity is shown as cisplatin IC $\mathbf{C}_{50}$ ( $(\mathbf{D}$ and $\mathbf{E})$ Restoration of decreased cell viability and cisplatin resistance by cell-permeable IP4 in ITPKB-knockdown cells. ITPKB-knockdown cells were treated in the presence and absence of $1 \mu \mathrm{M}$ of cell-permeable IP4 (IP4-PM). Cell viability (D) and cisplatin IC $\mathbf{C}_{50}(\mathbf{E})$ were determined as described in B and C. (F) Intracellular level of IP4 in KB-3-7 ${ }^{\text {cisR }}$ cells with ITPKB variants was obtained by ${ }^{31} \mathrm{P}$ nuclear magnetic resonance $\left({ }^{31} \mathrm{PNMR}\right)$ spectroscopy. IP4 NMR spectra and concentrations in reaction buffer $(\mu \mathrm{M})$ are shown. Data are mean \pm SD from 3 technical replicates of each sample for $\mathbf{A}-\mathbf{E}$ and are representative of $4(\mathbf{A})$ and $3(\mathbf{B}-\mathbf{E})$ independent biological experiments. Statistical analysis was performed by 1 -way ANOVA (**** $P<0.0001)$.

patient tumors and HNSCC, lung, and ovarian cancer PDX tumors were studied is provided in Supplemental Tables 1 and 2. These data demonstrate that ITPKB expression pattern positively correlates with cancer cisplatin resistance.

ITPKB is important for cisplatin-resistant cancer cell proliferation and tumor growth. To investigate the role of ITPKB in cancer cell growth in the presence of cisplatin, we target-downregulated ITPKB using 2 distinct shRNA clones in KB-3-1 ${ }^{\text {cisR }}$, A549 $9^{\text {cisR }}$, and A2780 $0^{\text {cisR }}$ cells. Knockdown of ITPKB significantly attenuated viability of the cells and decreased colony-forming potential only in the presence of cisplatin (Figure 2A and Supplemental
Figure 2A). In addition, ITPKB knockdown sensitized the cells to cisplatin treatment as shown by the cisplatin $\mathrm{IC}_{50}$ (Figure 2B). Similar results were obtained by ITPKB knockout using 2 distinct sgRNA clones (Supplemental Figure 2, B and C). Next, we validated the role of ITPKB in vivo in a xenograft mouse model. Tumors originating from KB-3-1 ${ }^{\text {cisR }}$ cells with ITPKB knockdown showed an apparent decrease in tumor growth and tumor size in mice treated with cisplatin (Figure $2 \mathrm{C}$ and Supplemental Figure 2D). These data reveal that ITPKB promotes cisplatin-resistant potential and that targeting ITPKB sensitizes cisplatin-resistant cancer cells to cisplatin. 
A
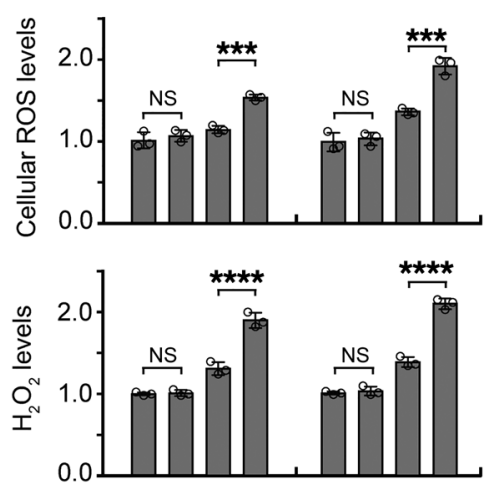

ITPKB shRNA - + $-+\quad+\quad+\quad-\quad+$

Cisplatin $\frac{-++}{\text { KB-3-1 cisR }} \frac{-++}{A 549 \text { cisR }}$

D

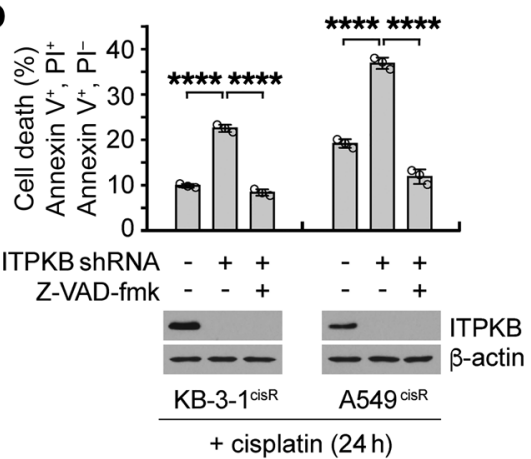

$\mathbf{F}$

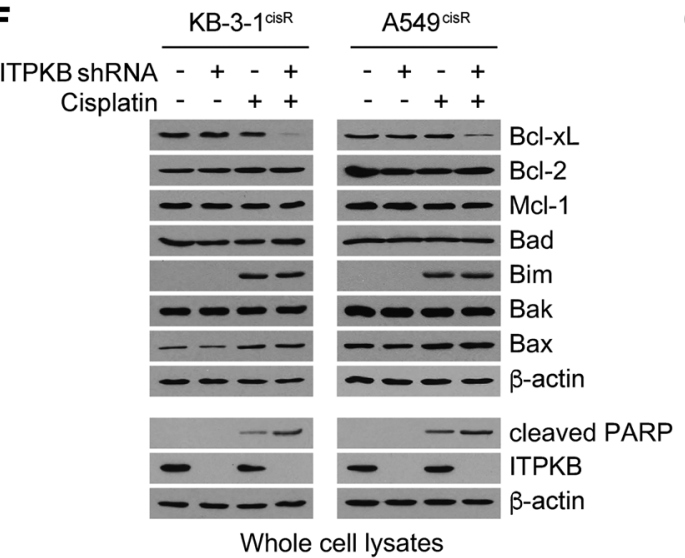

B
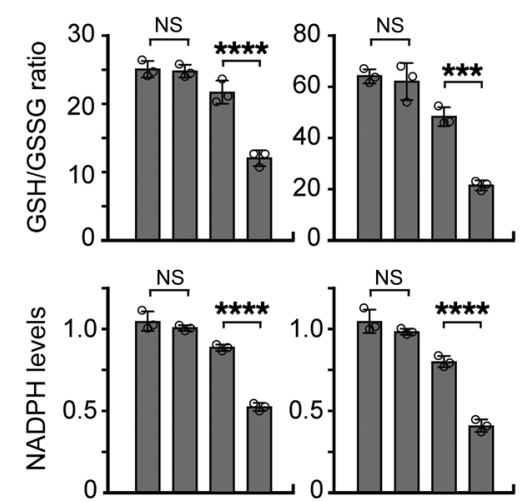

ITPKB shRNA - + - +

Cisplatin - -++

KB-3-1 ${ }^{\text {cisR }}$

E

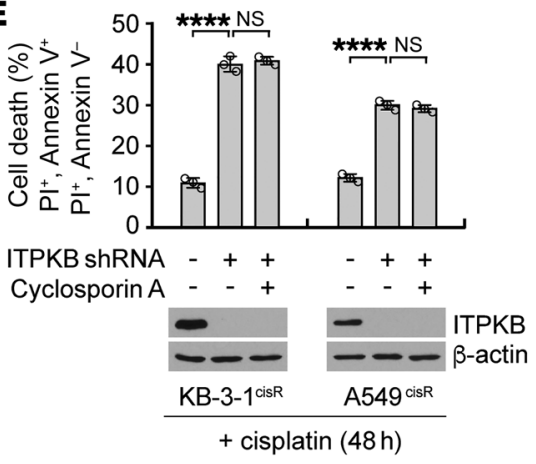

G

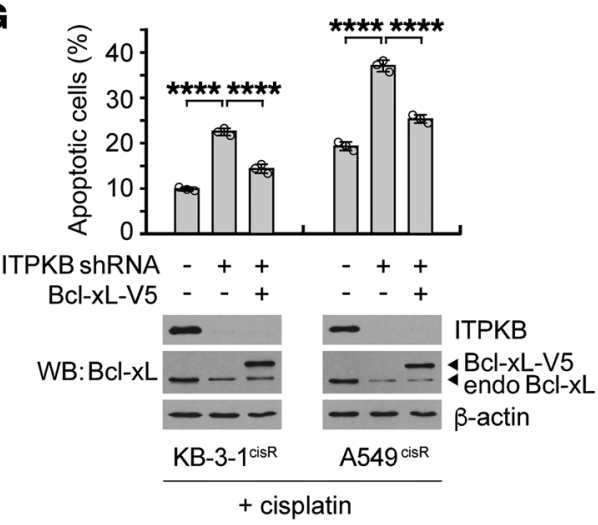

C
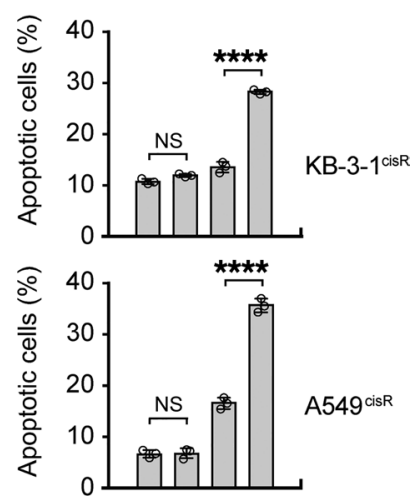

ITPKB shRNA - + - +

Cisplatin - - +

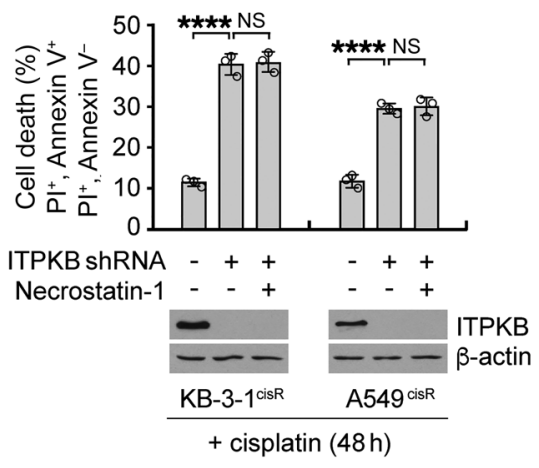

H
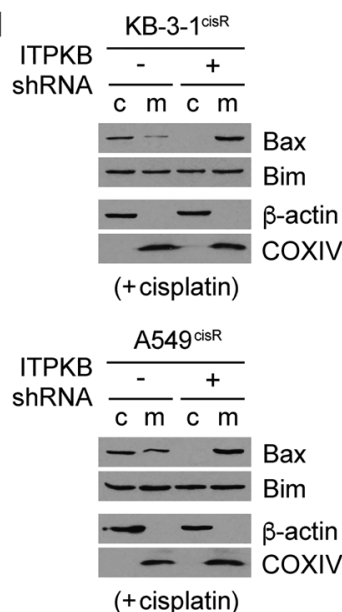

Figure 4. ITPIKB contributes to cisplatin resistance by attenuating ROS and suppressing apoptosis. (A and B) Effect of ITPKB knockdown and cisplatin treatment on redox status. (C) Effect of targeting ITPKB on apoptotic cell death in cells with ITPKB knockdown and cisplatin treatment. (D and E) Effect of apoptosis (D) or necrosis (E) inhibitors on cell death induced by ITPKB knockdown and cisplatin treatment. Cells were treated with Z-VAD-fmk (25 $\mu$ M), cyclosporin $A(100 \mathrm{nM})$, or necrostatin-1 $(20 \mu \mathrm{M})$, and apoptotic or necrotic cell death was monitored by annexin $V$ and propidium iodide (PI) staining. (F) Level of apoptosis-related factors in ITPKB-knockdown and cisplatin-treated cells was assessed by Western blotting. (G) Effect of Bcl-xL overexpression on apoptosis in ITPKB-knockdown and cisplatin-treated cells. (H) Bax mitochondrial translocation upon ITPKB knockdown in cisplatin-treated cells. c, cytosol; m, mitochondria. Data are mean \pm SD from 3 technical replicates of each sample. Data shown are representative of $3(\mathbf{A}-\mathbf{E}, \mathbf{G}$, and $\mathbf{H})$ and 2 (F) independent biological experiments. Statistical analysis was performed by 1 -way ANOVA $\left({ }^{* *} P<0.005 ;{ }^{* * *} P<0.0001\right)$.

IP4, the metabolite product of ITPKB, is responsible for cisplatin resistance. We next investigated whether ITPKB enzyme activity is important for cisplatin resistance. We produced a kinase-dead mutant form of ITPKB, D897N, in which the kinase activity that phosphorylates IP3 is lost (Figure 3A). Rescue expression of shR-
NA-resistant WT ITPKB but not the kinase-dead mutant (DN) ITPKB restored the decreased cell viability and cisplatin resistance mediated by ITPKB knockdown in cisplatin-resistant cancer cells in the presence of cisplatin (Figure 3, B and C). In addition, treatment with cell-permeable IP4, the metabolite product 
A
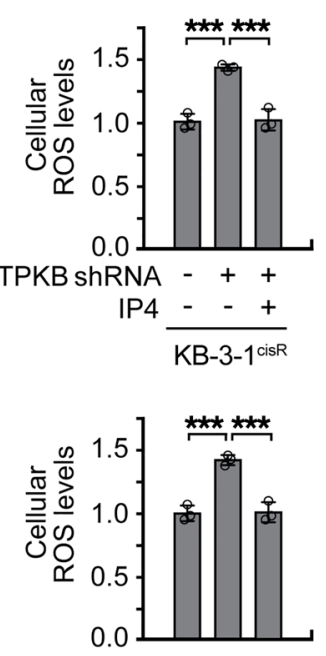

ITPKB ShRNA - + +

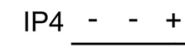

A549

+ cisplatin
B
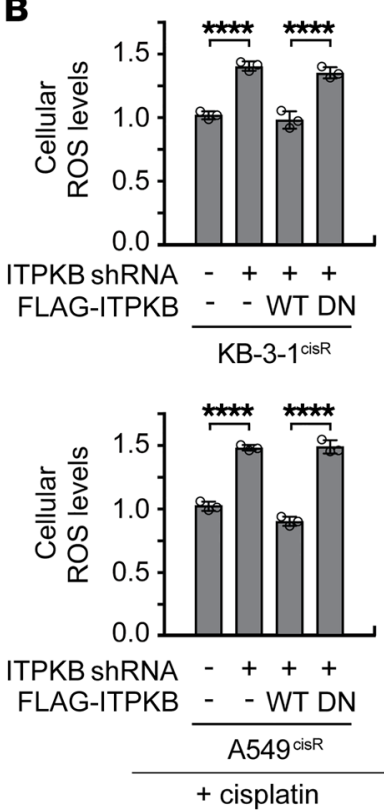

E

C

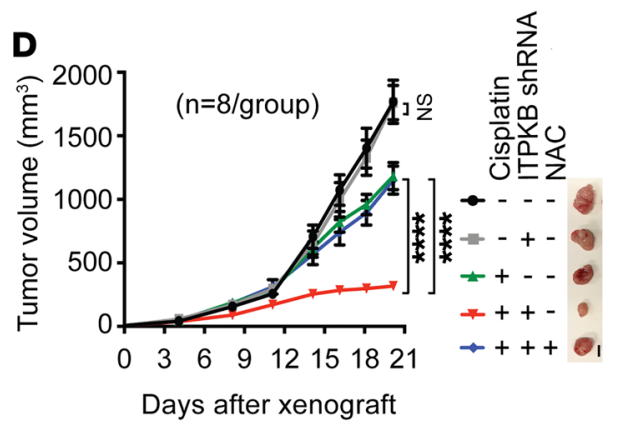

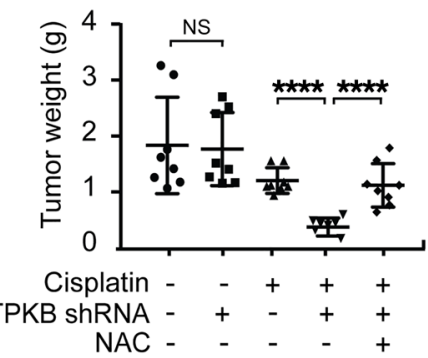

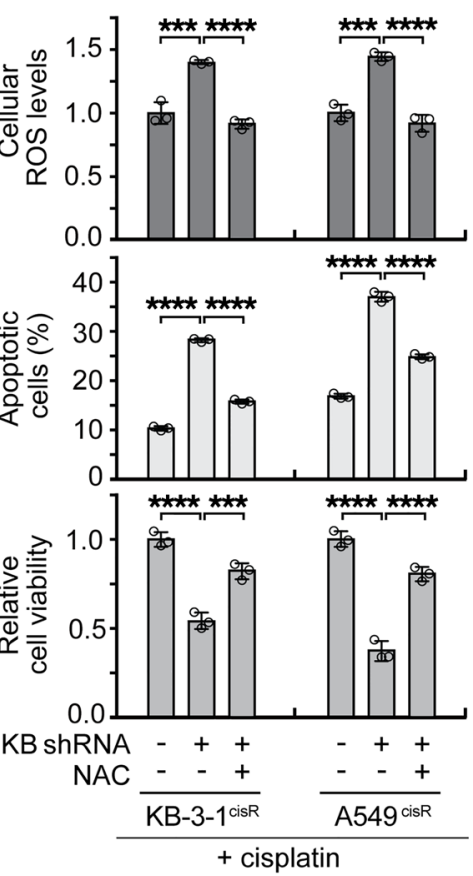

$\mathbf{F}$

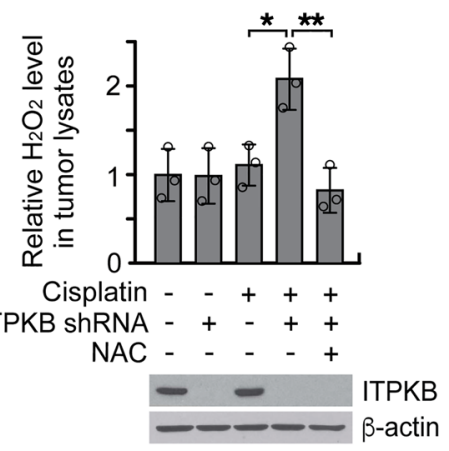

KB-3-1 ${ }^{\text {iss }}$ tumor lysates

Figure 5. Targeting ITPKB leads to cisplatin-induced ROS accumulation and rebalanced redox attenuates apoptosis and restores cisplatin-resistant tumor growth. (A and B) Intracellular ROS levels in cisplatin-resistant cells incubated with $1 \mu$ M IP4-PM (A) or with rescue expression of shRNA-resistant ITPKB WT or kinase-dead mutant (DN: D897N) (B) in the presence of cisplatin. (C) Effect of antioxidant NAC on cellular ROS level (top), apoptotic cell death (middle), and cell viability (bottom) of cisplatin-resistant cells with ITPKB knockdown and cisplatin treatment. Cells were treated with 0.5 mM NAC and cisplatin (KB-3-1 ${ }^{\text {cisR }}, 5 \mu \mathrm{g} / \mathrm{ml}$; A549 ${ }^{\text {cisR }}, 2 \mu \mathrm{g} / \mathrm{ml}$ ), and ROS levels and apoptosis/cell viability were measured after 12 and 48 hours, respectively. (D-F) Rescue effect of NAC on tumor growth of xenograft mice bearing ITPKB-knockdown KB-3-1 $1^{\text {cisR }}$ cells. Mice were treated with PBS, cisplatin (5 mg/kg i.p. twice per week), or NAC ( $10 \mathrm{mg} / \mathrm{ml}$ in drinking water) 3 days after xenograft, and tumor size was measured (D). Tumor weight (E) and $\mathrm{H}_{2} \mathrm{O}_{2}$ level and ITPKB knockdown efficiency in tumors (F) are shown. Scale bars: $10 \mathrm{~mm}$ for $\mathbf{D}$. Error bars represent SEM for $\mathbf{D}$ and SD for $\mathbf{E}(n=8)$. Data are mean \pm SD from 3 technical replicates of each sample for $\mathbf{A}-\mathbf{C}$ and $\mathbf{F}$ and are representative of $2(\mathbf{A}, \mathbf{B}$, and $\mathbf{F})$ and $3(\mathbf{C})$ independent biological experiments. Statistical analysis was performed by 2-way ANOVA for $\mathbf{D}$ and 1-way ANOVA for all other data $\left({ }^{*} P<0.05 ;{ }^{* *} P<0.01 ;{ }^{* * *} P<0.005 ;{ }^{* * *} P<0.0001\right)$.

of ITPKB, partially but significantly restored the attenuated cell growth and cisplatin resistance of ITPKB-knockdown cells (Figure 3, $\mathrm{D}$ and $\mathrm{E}$ ). To further validate whether target downregulation of ITPKB attenuates IP4 levels in cells and whether the addition of WT ITPKB or cell-permeable IP4 restores the decreased intracellular IP4 levels and controls cisplatin resistance, we measured IP4 levels in cells that harbor ITPKB variants by nuclear magnetic resonance (NMR). Knockdown of ITPKB abolished IP4 levels in cells, while expression of WT ITPKB or treatment with exogenous IP4 rescued the decreased IP4 levels in ITPKB knockdown cells (Figure 3F). These data suggest that IP4, the metabolite product of ITPKB, is the main factor that confers resistance to cisplatin.
ITPKB promotes cisplatin resistance by controlling cisplatininduced ROS and apoptotic cell death. To explore whether ITPKB provides a metabolic advantage for cisplatin resistance, we performed a number of metabolic assays and investigated changes in bioenergetics, biosynthesis, and redox status upon ITPKB knockdown and cisplatin treatment. Attenuation of ITPKB and cisplatin treatment in cisplatin-resistant cancer cells did not affect intracellular energy levels, glycolytic rates, or biosynthesis of RNA and lipid (Supplemental Figure 3, A and B). However, we found that knockdown of ITPKB and cisplatin treatment resulted in enhanced intracellular ROS and $\mathrm{H}_{2} \mathrm{O}_{2}$ levels (Figure 4A). In line with this observation, antioxidant readouts including 


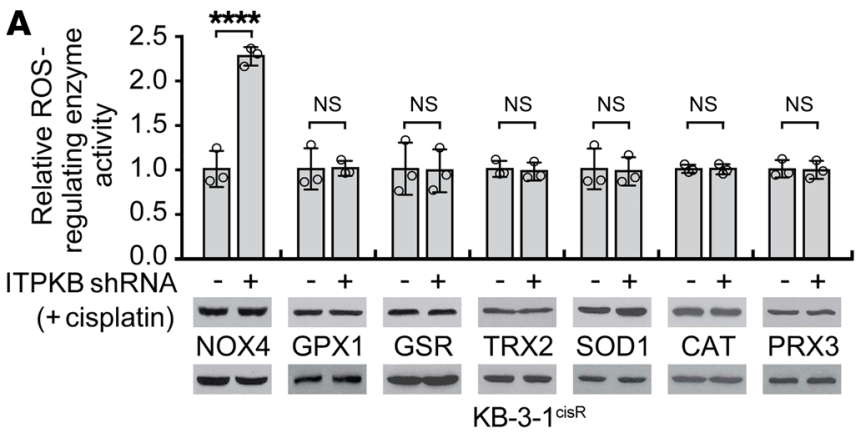

B

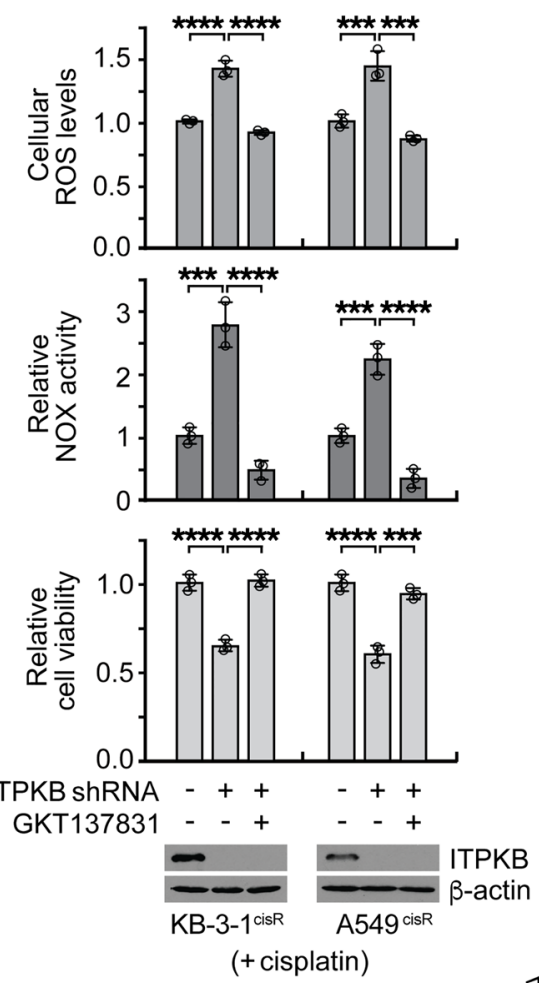

C
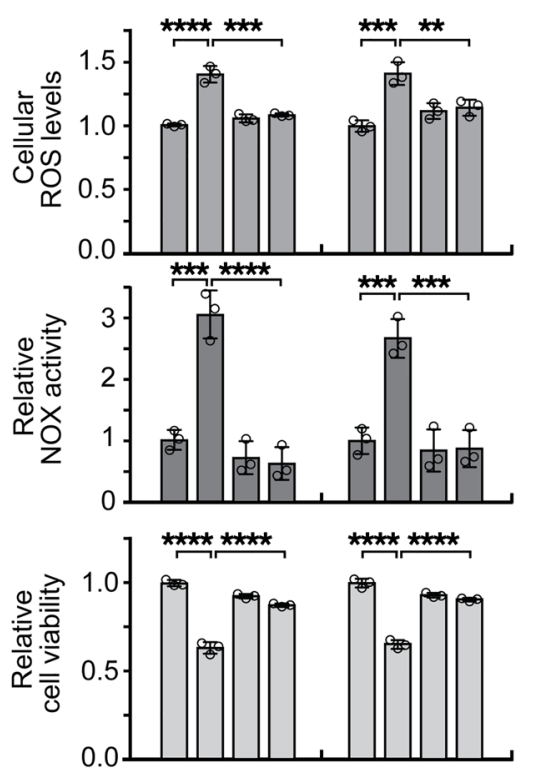

ITPKB ShRNA - + + + + + + NOX4 ShRNA - - \#1 \#2 - - \#1 \#2

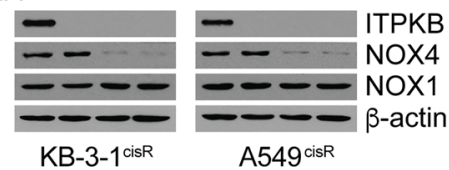

(+ cisplatin)

G

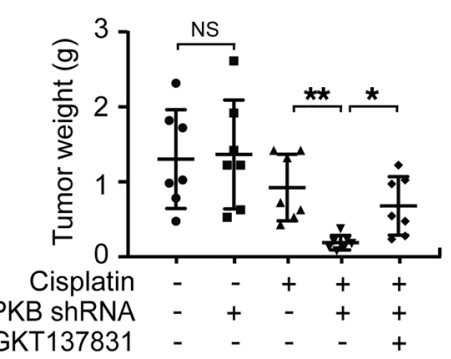

D

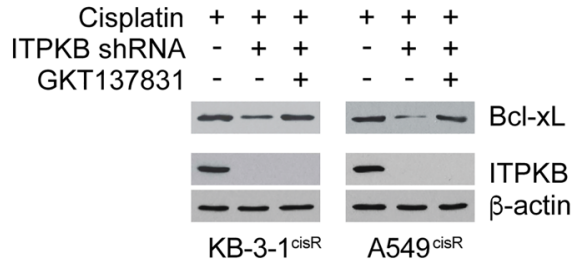

E

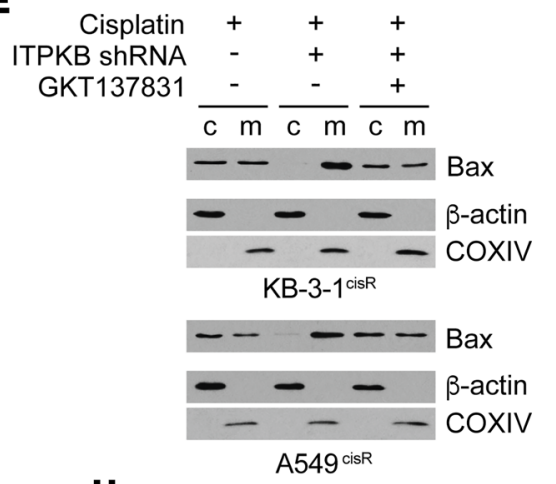

H
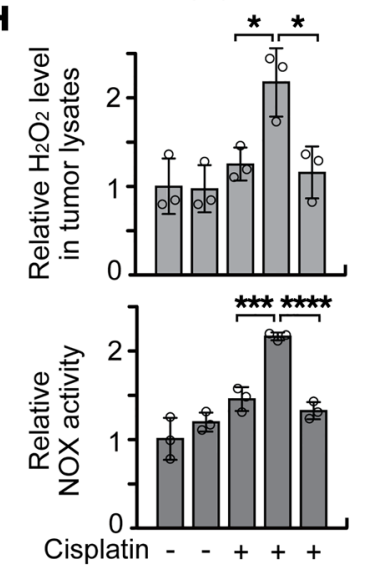

ITPKB shRNA - + - + +

GKT137831 - - - - +

ITPKB

$-\beta$-actin

KB-3-1 ${ }^{\text {cisR }}$ tumor lysates

Figure 6. ITPKB contributes to cisplatin resistance by dampening NOX4 activity in cancer cells. (A) Effect of ITPKB knockdown on the activity of

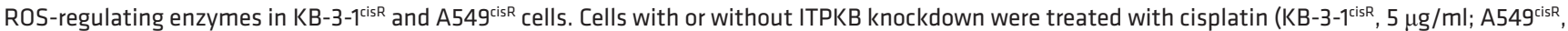
$2 \mu \mathrm{g} / \mathrm{ml}$ ), and activities of redox regulatory enzymes were measured after 12 hours. (B) Effect of NOX1/4 inhibitor GKT137831 on ROS level, NOX activity, and cell viability in ITPKB-knockdown cells in the presence of cisplatin. Cells were treated with CKT137831 (10 $\mu$ M) and cisplatin as in A. (C) Effect of NOX4 knockdown on ROS level, NOX activity, and cell viability in ITPKB-knockdown cells in the presence of cisplatin. (D and E) Bcl-xL expression (D) and Bax localization (E) change upon GKT137831 treatment in ITPKB-knockdown cells. c, cytosol; m, mitochondria. (F-H) The rescue effect of NOX inhibitor on the tumor growth of ITPKB-knockdown KB-3- c $^{\text {isR }}$ xenograft mice treated with cisplatin. Mice were treated with vehicle control, cisplatin (5 mg/kg), and GKT137831 (30 mg/kg) by i.p. injection twice a week from 3 days after xenograft. Tumor size (F), tumor weight (G), $\mathrm{H}_{2} \mathrm{O}_{2}$ level (H, top), NOX activity (H, middle), and ITPKB expression in tumors (H, bottom) are shown. Scale bars: $10 \mathrm{~mm}$ for $\mathbf{F}$. Error bars represent SEM for $\mathbf{F}$ and SD for $\mathbf{G}(n=7)$. Data are mean \pm SD from 3 technical replicates of each sample and are representative of 2 independent biological experiments for $\mathbf{A}-\mathbf{E}$ and $\mathbf{H}$. Statistical analysis was performed by 2-way ANOVA for $\mathbf{F}$ and 1-way ANOVA for all other data $\left({ }^{*} P<0.05 ;{ }^{* *} P<0.01 ;{ }^{* *} P<0.005 ;{ }^{* * *} P<0.0001\right)$. 
A

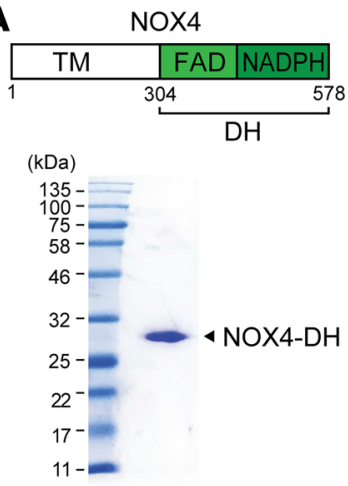

B

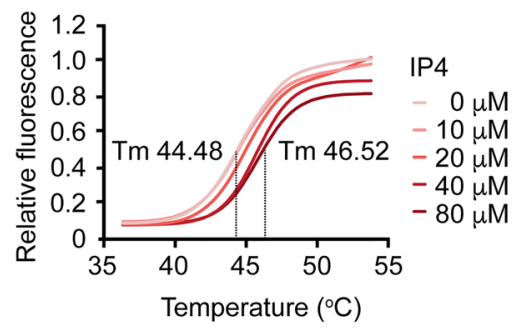

C

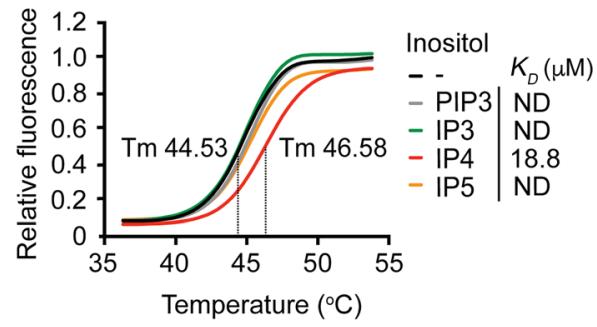

D

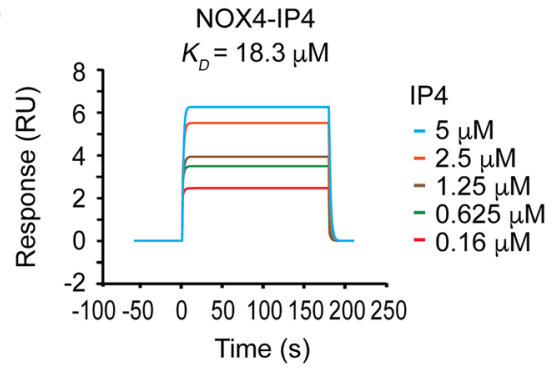

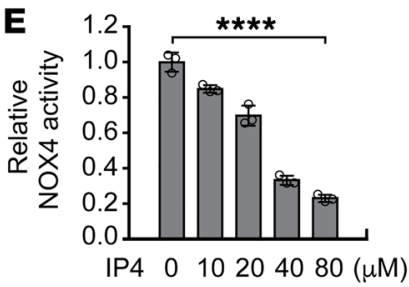

WB:FLAG $-\infty-\infty$

FLAG-NOX4-DH

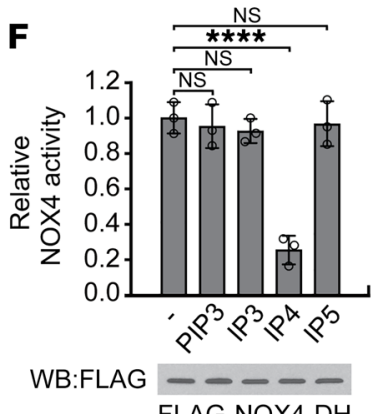

FLAG-NOX4-DH

G

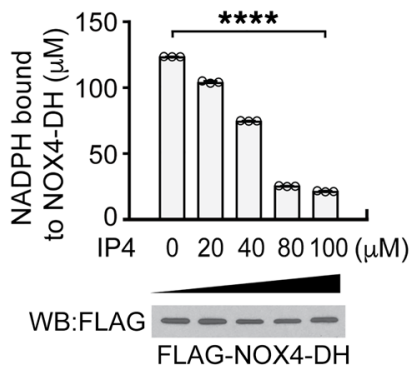

Figure 7. The ITPKB product IP4 directly binds to NOX4 and suppresses its activity by competing with the cofactor NADPH for binding. (A) Purification of the recombinant NOX4 dehydrogenase active domain (NOX4-DH). Top: Schematic representation of NOX4 domains. TM, transmembrane domain; FAD, flavin adenine dinucleotide binding domain; NADPH, NADPH binding domain. Bottom: Coomassie staining of purified recombinant NOX4-DH. (B) Thermal shift analyses of NOX4-DH incubated with increasing concentrations of IP4. Tm, melting temperature. (C) Thermal shift analyses of NOX4-DH incubated with $80 \mu \mathrm{M}$ of the inositol metabolites PIP3, IP3, IP4, and IP5. Dissociation constant $\left(K_{D}\right)$ values for the interaction are shown. ND, not determined. (D) Interaction between IP4 and NOX4-DH was determined by Biacore surface plasmon resonance analysis and is shown as a $K_{0}$ value. (E and F) The activity of purified NOX4-DH was measured in the presence of increasing concentrations of IP4 (E) or $80 \mu \mathrm{M}$ of different inositol metabolites (F). (G) Quantification of NADPH bound to NOX4 in the presence of increasing concentrations of IP4. FLAG-tagged NOX4-DH (30 $\mu$ M) was incubated with NADPH (100 $\mu$ M) and IP4 $(0-100 \mu \mathrm{M})$. Amount of NADPH retained on immobilized NOX4-DH was determined by measurement of absorbance at A340 nm. Data are mean \pm SD from 3 technical replicates of each sample for $\mathbf{E}-\mathbf{C}$. Data shown are representative of 2 (B-D) and $3(\mathbf{E}-\mathbf{G})$ independent biological experiments. Statistical analysis was performed by 1 -way ANOVA $(* * * * 0.0001)$.

reduced/oxidized glutathione (GSH/GSSG) ratio and NADPH levels were significantly decreased (Figure 4B). These data suggest that ITPKB is critical for regulating redox status, particularly in regulating cisplatin-induced ROS.

To further examine whether ITPKB is involved in steps during the binding of cisplatin to DNA and cisplatin-mediated DNA damage, we measured cisplatin-DNA adducts and phosphorylation of $\gamma \mathrm{H} 2 \mathrm{AX}$, respectively, in ITPKB-knockdown cells treated with cisplatin. Knockdown of ITPKB had no impact on cisplatin-induced DNA adducts or cisplatin-induced DNA damage, suggesting that the effect of ITPKB on cisplatin resistance occurs through neither pretarget nor on-target resistance, but through a post-target resistance mechanism (Supplemental Figure 3, C and D). Indeed, loss of ITPKB significantly sensitized cells to cisplatin-induced apoptotic cell death (Figure 4C). Together, these data suggest that ITPKB confers cisplatin-resistant cell survival by controlling cisplatin-induced ROS and consequently suppressing cell death induction. Our data suggest that the cell death induced by ITPKB loss and cisplatin treatment was apoptosis rather than necrosis, since treatment with a pan-caspase inhibitor, Z-VAD-fmk, but not cyclosporin A or necrostatin-1, which block necrosis, reversed the cell death induced by loss of ITPKB and cisplatin treatment (Figure 4, D and E). To further decipher the downstream mechanism by which the combination of ITPKB knockdown and cisplatin treatment increases apoptotic cell death, we assessed the level of apoptotic factors. ITPKB knockdown and cisplatin treatment specifically resulted in the attenuation of Bcl-xL (Figure $4 \mathrm{~F}$ ). In contrast, restoration of decreased Bcl-xL by overexpression significantly reduced apoptosis mediated by ITPKB 
A

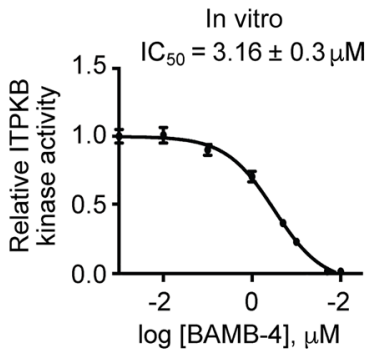

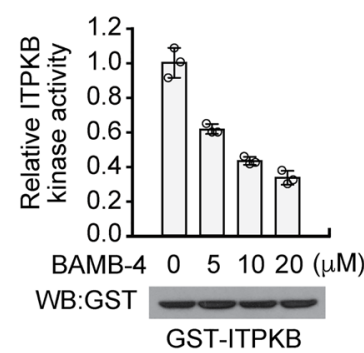

GST-ITPKB
C

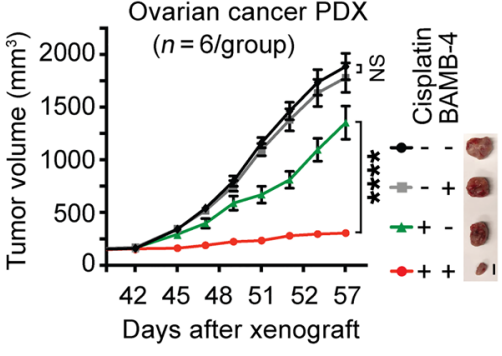

D

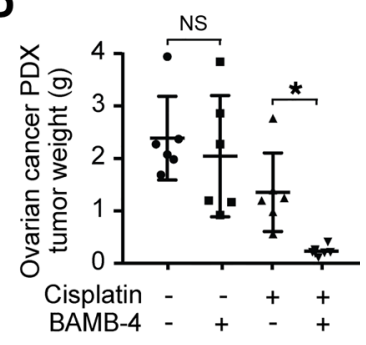

B
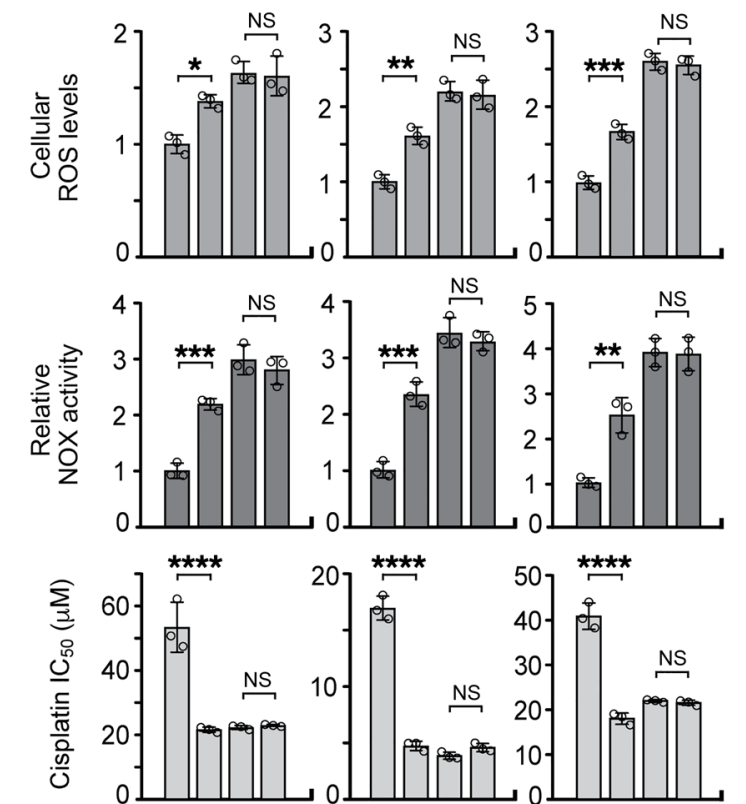

ITPKB sgRNA - - + +

BAMB-4 $(10 \mu \mathrm{M})-++$

Cisplatin ++++

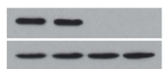

KB-3-1 ${ }^{\text {cisR }}$

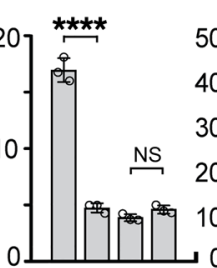

$-++$

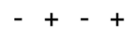

$+++$

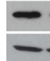

A549 cisR

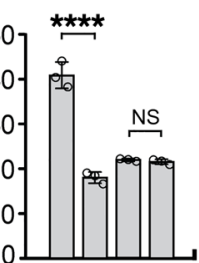

$-++$

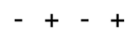

$+++$

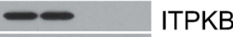

- - - - -actin A2780 cisR
E

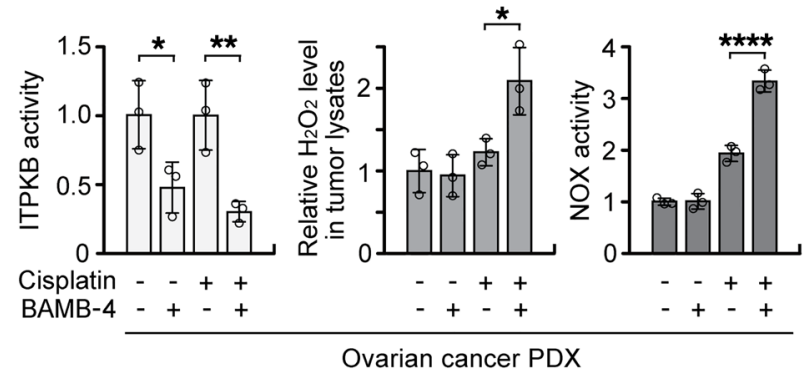

$\mathbf{F}$

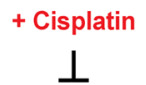

DNA synthesis

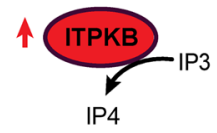

Cisplatin-resistant cancer cells

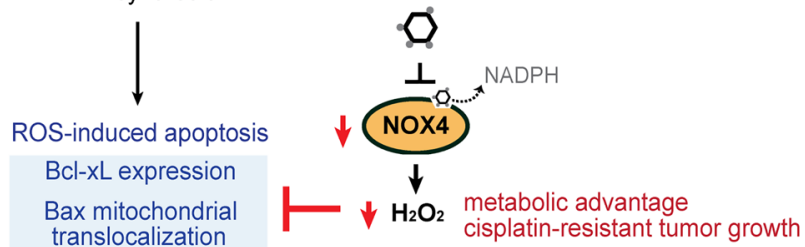

Figure 8. Pharmacological inhibition of ITPKB sensitizes cancer cells to cisplatin treatment resulting in attenuated tumor growth in PDX mouse models.

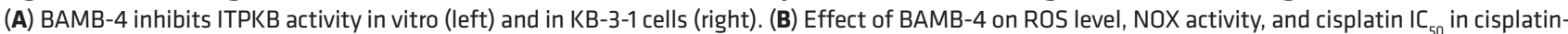

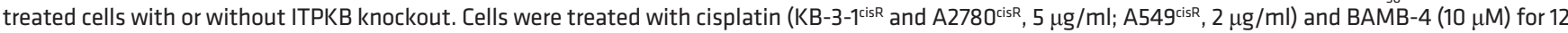
hours, and ROS and NOX activity were examined. (C and D) Effect of BAMB-4 and/or cisplatin treatment on tumor growth (C) and tumor size (D) of ovarian cancer PDX mice. Mice were treated with cisplatin ( $5 \mathrm{mg} / \mathrm{kg})$ and BAMB-4 (10 mg/kg) by i.p. injection twice a week after 42 days from xenograft. (E) Effect of BAMB-4 and/or cisplatin treatment on ITPKB activity, $\mathrm{H}_{2} \mathrm{O}_{2}$ level, and NOX activity in ovarian cancer PDX tumors is shown. Scale bars: 10 mm for $\mathbf{C}$. Error bars represent SEM for $\mathbf{C}$ and SD for $\mathbf{D}(n=6)$. Data are mean \pm SD from 3 technical replicates of each sample and are representative of 2 independent biological experiments for A, B, and E. Statistical analysis was performed by 2 -way ANOVA for $\mathbf{C}$ and 1 -way ANOVA for all other data $\left({ }^{*} P<0.05\right.$; ${ }^{* *} P<0.01 ;{ }^{* *} P<0.005$; $\left.{ }^{* * * *} P<0.0001\right)$. (F) Proposed model of ITPKB-mediated cisplatin resistance in human cancer. The ITPKB product IP4 inhibits NOX4 by competing with its cofactor NADPH for binding. IP4-induced NOX4 inhibition controls cisplatin-induced ROS, which provides a metabolic advantage to cancer cells that leads to cisplatin-resistant cancer cell survival and tumor growth.

knockdown and cisplatin treatment (Figure 4G). Bcl-xL is known to prevent the mitochondrial translocation of Bax, a critical event during apoptosis (32). Indeed, ITPKB knockdown and cisplatin treatment resulted in translocation of Bax to mitochondria (Figure $4 \mathrm{H})$. These data suggest that ITPKB manages cisplatin-induced ROS and prevents cells from undergoing apoptotic cell death by maintaining antiapoptotic Bcl-xL and preventing Bax activation.

Consistent with previous observations in other cell types, impairment of ITPKB was accompanied by increased AKT signaling but no change in the MAPK pathway regardless of cisplatin treatment in KB-3-1 $1^{\text {cisR }}$ and A549 $9^{\text {cisR }}$ cells (Supplemental Figure 3E). Nevertheless, since ITPKB knockdown enhanced cisplatin-induced apoptosis and attenuated cell growth, it is anticipated that AKT is not part of the ITPKB-mediated cisplatin-resistant signaling axis and elevation of AKT signaling upon ITPKB knockdown does not compensate for the prosurvival potential lost in cisplatin-treated ITPKB-knockdown cells.

We next investigated whether the ITPKB product, IP4, contributes to the cisplatin-mediated elevation of ROS levels. Rescue of reduced intracellular IP4 levels by the cell-permeable form of 
IP4 or overexpression of WT ITPKB but not kinase-dead mutant D897N reduced the elevated ROS levels in ITPKB-knockdown cells, suggesting that the enzyme activity of ITPKB and its product IP4 is required to control ROS in cisplatin-resistant cancer cells (Figure 5, A and B). Next, we further confirmed whether ITPKB promotes cisplatin-resistant cell survival and tumor growth through redox regulation using antioxidant $\mathrm{N}$-acetylcysteine (NAC). Indeed, treatment with NAC significantly reduced cisplatin-induced ROS levels and apoptotic cell death, and rescued cell viability in ITPKB-knockdown cells (Figure 5C). ROS scavenged by ITPKB and its product IP 4 were nonmitochondrial, as neither ITPKB loss nor extracellular IP4 altered the levels of cisplatin-induced mitochondrial ROS (Supplemental Figure $4 \mathrm{~A})$. Overexpression of catalase, a cytoplasmic hydrogen peroxide scavenger, but not mitochondria-targeted antioxidant mito-TEMPO (Sigma-Aldrich) reversed the increased apoptosis and impaired cell viability induced by ITPKB knockdown and cisplatin treatment. This suggests that ITPKB contributes to cisplatin-resistant cell survival by removing cytosolic hydrogen peroxide rather than mitochondrial ROS (Supplemental Figure 4, $\mathrm{B}$ and $\mathrm{C}$ ). In line with the in vitro observations, NAC treatment diminished cisplatin-induced ROS levels and rescued the tumor

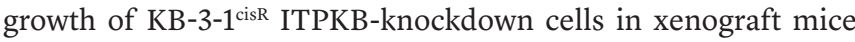
(Figure 5, D-F, and Supplemental Figure 4D).

ITPKB controls cisplatin-induced ROS by inhibiting the ROSproducing enzyme NOX4. To explore how ITPKB regulates the levels of cisplatin-induced ROS in cancer cells, we examined whether knockdown of ITPKB alters the activity of enzymes involved in ROS regulation. These enzymes include NADPH oxidase (NOX), glutathione peroxidase (GPX), glutathione reductase (GSR), thioredoxin (TRX), superoxide dismutase (SOD), catalase (CAT), and peroxiredoxin (PRX). Only activity of $\mathrm{NOX}$, which produces $\mathrm{H}_{2} \mathrm{O}_{2}$, was significantly increased in cisplatin-treated cells that lack ITPKB (Figure 6A). Similar results were obtained in ITPKB-knockout cells. Knockout of ITPKB enhanced NOX activity and ROS and induced apoptosis in the presence of cisplatin (Supplemental Figure 5A). To further substantiate that ITPKB controls cisplatin-induced ROS by inhibiting NOX, we showed that inhibition of NOX by the NOX1/4 inhibitor GKT137831 decreased the enhanced ROS level and NOX activity, and rescued the reduced cell viability (Figure 6B). Interestingly, ITPKB knockdown enhanced NOX activity, ROS levels, and sensitivity to other DNA-damaging agents such as mitomycin $\mathrm{C}$ or doxorubicin but not to paclitaxel, which induces cell death through chromosome missegregation (Supplemental Figure 5B). Moreover, NOX4 but not NOX1 knockdown mimicked the effects of NOX inhibitor, resulting in reduced ROS and NOX activity as well as restoration of attenuated cell viability in ITPKB-knockdown cells treated with cisplatin (Figure 6C and Supplemental Figure 5C). NOX4, ITPKB, and IP4 predominantly exist in the cytosol, and cytosolic but not mitochondrial NOX activity was enhanced by ITPKB loss and cisplatin treatment, suggesting that ITPKB controls NOX4 activity in the cytosol (Supplemental Figure 6, A-D). Moreover, inhibition of NOX4 restored the decreased $\mathrm{Bcl}-\mathrm{xL}$ expression and retrotranslocated Bax from the mitochondria into the cytosol in cisplatin-treated ITPKB-knockdown cells, suggesting that ITPKB manages cisplatininduced ROS and prevents cells from undergoing apoptotic cell death through inhibiting NOX4 (Figure 6, D and E). The ITPKB/
NOX4 signaling axis was validated in vivo in xenograft mice. Mice bearing tumors with ITPKB knockdown and treated with cisplatin had significantly smaller tumors than control groups with ITPKB knockdown or cisplatin treatment alone, whereas inhibition of NOX significantly reversed the decreased tumor growth (Figure $6 \mathrm{~F}$ ), tumor weight (Figure 6G), enhanced ROS and NOX activity (Figure $6 \mathrm{H}$ ), and tumor proliferation (Supplemental Figure 6E) of these cisplatin-treated ITPKB-knockdown tumors. These data together provide evidence that ITPKB signals through IP4 to inhibit NOX4 and control ROS and obtain resistant potential to DNA-damaging agents in cancer cells.

To explore the molecular mechanism by which ITPKB inhibits NOX4 activity, recombinant dehydrogenase domain of NOX4 (NOX4-DH) was purified (Figure 7A). Since our study demonstrates that the ITPKB product IP4 controls cisplatininduced ROS, we hypothesized that IP4 binds to and inhibits NOX4. Thermal shift assay of recombinant NOX4-DH with increasing concentrations of IP4 revealed a direct interaction between NOX4 and IP4 (Figure 7B). The $K_{D}$ value of the NOX4IP4 interaction was calculated to be $18.80 \pm 6.35 \mu \mathrm{M}$, whereas $K_{D}$ values of the other inositol derivatives and NOX4 interaction were not determined. Incubation of NOX4 with various inositol metabolites, including PIP3, IP3, IP4, and IP5, indicated that NOX4 specifically binds to IP4 but no other inositol derivatives (Figure 7C). In addition, surface plasmon resonance-based kinetic analysis of IP 4 and NOX 4 showed that IP 4 directly binds to NOX4 (Figure 7D). We observed that IP4 but not the other inositol derivatives interfered with NOX4 activity in vitro (Figure 7, E and F). Furthermore, IP4 competed with NADPH in binding NOX4 (Figure 7G). This suggests that IP4, the metabolite product of ITPKB, inhibits NOX4 by interfering in the binding of NOX4 to its cofactor NADPH.

Both initial and cisplatin-induced ITPKB contributes to cisplatin resistance. We next examined whether the ITPKB that confers cisplatin resistance is intrinsic or acquired. Although they differed in ITPKB level and NOX activity, both naive and cispatin-resistant cell lines responded to ITPKB knockdown, showing enhanced NOX activity and elevated ROS levels, suggesting that ITPKB controls NOX and ROS in both parental and cispatin-resistant cells (Supplemental Figure 7, A-D). Moreover, modulation of ITPKB level by knockdown or overexpression altered cisplatin resistance in parental cells, suggesting that innate ITPKB contributes to cisplatin resistance (Supplemental Figure 7, E and F). We also compared ITPKB expression in pre- and post-treatment paired tumor samples obtained from individual HNSCC patients who received platinum-containing chemotherapy. Levels of ITPKB were significantly increased after treatment (Supplemental Figure 8, A and B). Patients who did not respond to therapy showed significantly greater ITPKB levels compared with the group who responded to treatment in tumors collected both before and after platinum treatment (Supplemental Figure 8C). Collectively, these results indicate that the initial ITPKB level contributes to cisplatin resistance while ITPKB is further induced during platinum treatment, and both initial and cisplatin-induced ITPKB contribute to cisplatin-resistant tumor growth.

Pharmacological inhibition of ITPKB sensitizes cancer cells to cisplatin in vitro and in vivo. Our finding that ITPKB is abundant in cisplatin-resistant tumors and that genetic loss of ITPKB refines 
cisplatin response indicates that ITPKB could be a promising anticancer target for cisplatin-resistant cancers. Therefore, we tested the efficacy of pharmacologically targeting ITPKB on cisplatin response in cancer cell lines and PDX mouse models. BAMB-4, a cell-permeable inhibitor of ITPK that competes with IP3 and ATP for binding, effectively inhibited the activity of ITPKB in vitro and in cancer cells (Figure 8A). Inhibition of ITPKB activity by BAMB-4 treatment resulted in increased ROS level, NOX activity, and cisplatin response in cancer cells. The BAMB- 4 effect was abolished in cells lacking ITPKB (Figure 8B and Supplemental Figure 9, A and B). However, removal of inositol polyphosphate multikinase (IPMK), another kinase that uses IP3 as a substrate, did not alter the effect of BAMB-4 on cisplatin sensitivity, suggesting that this effect occurs by targeting of ITPKB and not by targeting of another kinase such as IPMK (Supplemental Figure 9, C-E). Lastly, we evaluated the effect of pharmacological inhibition of ITPKB on cisplatin-resistant tumor growth in vivo in PDX mouse models of ovarian cancer and lung cancer. No obvious histopathological changes or kidney injury were observed in mice chronically exposed to BAMB-4 $(10 \mathrm{mg} / \mathrm{kg})$, cisplatin $(5 \mathrm{mg} / \mathrm{kg}$ ), or the combination (Supplemental Figure 10). The combination of BAMB-4 and cisplatin significantly reduced tumor growth, tumor size, and tumor proliferation in ovarian cancer PDX and lung cancer PDX mice (Figure 8, C and D, and Supplemental Figure 11, A-D). BAMB-4 successfully inhibited ITPKB activity and elevated $\mathrm{H}_{2} \mathrm{O}_{2}$ and NOX activity in PDX tumors (Figure 8E and Supplemental Figure 11E). These data provide proof of principle that ITPKB could serve as an effective synthetic lethal target partner with cisplatin in the treatment of cisplatin-resistant cancers.

\section{Discussion}

Emerging evidence indicates that altered cellular metabolism is a defining characteristic of nearly all types of cancers, and chemotherapy resistance presents a central obstacle in the majority of advanced cancers. However, how dysregulated tumor metabolism affects chemoresistance is poorly understood. Our findings suggest a mechanism involving crosstalk between cellular signaling-mediated redox metabolism and cisplatin resistance. We demonstrate that abundant ITPKB in cisplatin-resistant cancer cells metabolizes IP3 to IP4. The metabolite product IP4 binds to and inhibits NOX4 activity to produce the ROS hydrogen peroxide by interfering in the binding of its cofactor NADPH to NOX4. ITPKB-and IP4-mediated attenuation of NOX4 results in less ROS production upon cisplatin exposure, which contributes to cytoprotective antioxidation, leading to decreased ROS-mediated apoptotic cell death and consequently resulting in cisplatin-resistant tumor survival and growth (Figure $8 \mathrm{~F}$ ). Therefore, targeting ITPKB could be a therapeutic strategy to improve the efficacy of cisplatin-based chemotherapy in cancer treatment.

Although treatment with antioxidant NAC fully reversed the enhanced cellular ROS seen in ITPKB-target-downregulated cells treated with cisplatin, it partially but significantly rescued the enhanced apoptotic cell death and decreased cell viability induced by cisplatin and ITPKB knockdown (Figure 5C). This implies that there may exist an alternative role of ITPKB in cisplatin resistance in addition to controlling redox status, which could involve metabolism-independent signaling. Other chemotherapy agents, including paclitaxel, could provide oxidative stress to cancer cells, resulting in cell death (33). However, our data indicate that NOX4 regulation mediated by ITPKB specifically manages oxidative stress induced by DNA damaging agents. This suggests that different chemotherapy agents may produce different ROS and distinct effector molecules and mechanisms could be involved in acquisition of resistance in human cancers.

Mechanistically, we found that the metabolite product of ITPKB, IP4, inhibits NOX4 by interfering in the binding of cofactor NADPH to NOX4. We previously reported that metabolic intermediates such as $\alpha$-ketoglutarate and fumarate work as signaling effectors to enhance activity of calcium/calmodulin-dependent protein kinase kinase 2 (CamKK2) or the ROS-scavenging enzyme glutathione peroxidase 1 (GPX1) in human cancers $(34,35)$. Our finding that IP4 binds to and controls NOX 4 activity provides additional evidence that metabolites function as signaling molecules, allowing the connection between cellular signaling pathways and metabolic pathways. We provided evidence that IP4 binds to NOX4, while increasing concentrations of IP4 decrease NADPH binding to the dehydrogenase domain of NOX4, suggesting that NADPH binding to NOX4 is interrupted by IP4. Further studies are warranted to define the precise region in the NOX4 dehydrogenase domain that binds to IP4 and assess whether it directly competes with NADPH or allosterically alters the structure to hinder the interaction of NADPH with NOX4.

A moderate increase in ROS can promote cell proliferation, whereas excessive amounts of ROS can cause oxidative damage to proteins, lipids, and DNA, leading to cell death $(36,37)$. Therefore, maintaining ROS homeostasis is crucial for cell growth and survival. The NOX family of proteins is known as a source of ROS in cancer (38). Previous studies demonstrate that targeting NOX4 reduces cisplatin-related ROS production and protects pig kidney or mouse auditory cells from cytotoxic cell death $(18,39)$. Conversely, a study shows that targeting NOX4 in the presence of cisplatin further promotes apoptotic cell death in kidney cancer cells (40). This implies that the role of NOX4 may vary depending on factors including cell feature, cancer type, and discrete intracellular ROS levels.

Considering the clinical relevance of our study, we learned that the expression level of ITPKB positively correlates with cisplatin resistance in various cancer cell lines, patient-derived xenograft tumors, and primary cancer patient tumor tissues. These findings support that ITPKB could be used as a promising marker to predict the treatment efficacy of cisplatin-based chemotherapy. The correlation was greater in patient-derived tumors compared with cell lines. The correlation coefficient values ranged from 0.919 to 0.963 despite a smaller sample size compared with values in the cell lines studied. It is possible that cancer cell lines maintained in culture over a long period of time may have altered the original property of tumors. This further supports that intrinsic ITPKB levels in tumor cells adequately reflect the cisplatin response potential.

Lastly, targeting ITPKB using specific shRNA clones or an inhibitor effectively sensitized cells to cisplatin treatment in vitro and in vivo in patient-derived xenograft mouse models of lung cancer and ovarian cancer. These findings provide evidence that ITPKB could be a promising target for therapeutic intervention in combination with cisplatin-based chemotherapy. It is noted that ITPKB has alternative functions in differentiation and development of hematopoietic cells. Therefore, the development of an 
efficient drug delivery system to bring both cisplatin and ITPKB inhibitor directly and specifically to tumor tissues and pharmacological investigation to optimize the nontoxic dose range are further warranted. We identified NOX4 as a critical downstream effector of ITPKB that contributes to cisplatin-resistant cancer cell survival and tumor growth. Therefore, an efficient NOX4 modulator could be investigated as an alternative and additional therapeutic agent to overcome resistance to cisplatin in future cancer treatment approaches.

\section{Methods}

Reagents. shRNA lentiviral constructs targeting ITPKB, NOX4, NOX1, IPMK, and TRC Human Kinase shRNA Gene Family Library were purchased from GE Healthcare Dharmacon. sgRNA lentiviral constructs to target ITPKB were obtained from Genecopoeia. The shRNA sense strand sequences were ACAGCTATGGAAATTGACAAA (ITPKB shRNA clone 1), GCCTTCAGAGAGTTCACTAAA (ITPKB shRNA clone 2), CCCTCAACTTCTCAGTGAATT (NOX4 shRNA clone 1), CAGAGTTTACCCAGCACAAAT (NOX4 shRNA clone 2), CCGCACACTGAGAAAGCAATT (NOX1 shRNA clone 1), CCAAGGTTGTTATGCACCCAT (NOX1 shRNA clone 2), GCCCTGTATAATGGATGTAAA (IPMK shRNA clone 1), and GCAAGTTCATTACTCTTTGTT (IPMK shRNA clone 2). ITPKB sgRNA sequences were AGCCGAGTCGCTGTCCCCCG (ITPKB sgRNA clone 1) and CGGGGGCGTCTCGCTGCCAC (ITPKB sgRNA clone 2). Primers for ITPKB shRNA-resistant silent mutant and ITPKB kinase-dead D897N mutant were obtained from Integrated DNA Technologies. $\mathrm{Bt}_{2}$-Ins (1345) P4/PM was obtained from SiChem. GKT137831, Z-VAD-fmk, cyclosporin A, and necrostatin-1 were from Selleckchem. MitoSOX Red Mitochondrial Superoxide Indicator, MitoTracker Red, and Urea Nitrogen Detection kit were from Invitrogen. MitoPY1 was from Tocris Bioscience. Inositol derivatives were obtained from Cayman. BAMB-4 was purchased from Apexbio. Reagents for creatine and NAG activity assays were from Abcam. Mitochondria Isolation kit and BCA Protein Assay kit for proteinuria detection were from Pierce. Other chemicals were obtained from Sigma-Aldrich.

Antibodies. Antibodies against ITPKB (sc-11210/P-17), glutathione reductase (GSR) (sc-133245/C-10), thioredoxin 2 (TRX2) (sc-133201/F-10), superoxide dismutase 1 (SOD1) (sc-101523/24), catalase (sc-271358/A-4), Bcl-xL (sc-8392/H-5), and Mcl-1 (sc12756/22) were purchased from Santa Cruz Biotechnology. Antibodies against p-AKT Thr308 (13038/D25E6), AKT (4685/11E7), p-S6 ribosomal protein Ser240/Ser244 (5364/D68F8), S6 ribosomal protein (2217/5G10), p-MEK1/2 Ser221 (2338/166F8), MEK1/2 (9126/47E6), p-p44/42 MAPK (Erk1/2) Thr202/Tyr204 (4376/20G11), p44/42 MAPK (Erk1/2) (4695/137F5), p-histone $\gamma$ H2AX Ser139 (9718/20E3), myc (2276/9B11), cleaved PARP (5625/D64E10), Bcl-2 (2872), Bad (9239/D24A9), Bim (2933/ C34C5), Bak (12105/D4E4), Bax (5023/D2E11), and COX IV (4850/3E11) were obtained from Cell Signaling Technology. Antibodies against Ki-67 (ab92742/EPR3610), cisplatin-modified DNA (ab103261/CP9/19), glutathione peroxidase 1 (GPX1) (ab108427/ EPR3312), peroxiredoxin 3 (PRX3) (ab128953/EPR8115), IPMK (ab96753), and Kim1/Tim1 (ab47635) were obtained from Abcam. Anti-NOX4 antibodies were obtained from ProSci (7927) for immunoblotting cell lysates and Abcam (ab133303/UOTR1B493) for immunofluorescence staining and immunoblotting of recombinant
NOX4-DH. Anti-NOX1 antibody was from Novus Biologicals (NBP1-31546). Anti- $\beta$-actin antibody (A1978/AC-15), anti-FLAG antibodies (F1804/M2 and F7425), and anti-glutathione-S-transferase (GST) antibody (G1160/GST-2) were from Sigma-Aldrich.

Cell culture. 293T, Caov-3, BG-1, and KB-3-1 cells were cultured in DMEM with 10\% FBS. A2780, 1A9, A549, HCC827, H358, H1299, H460, H1975, Calu-1, and H157 cells were cultured in RPMI 1640 medium with $10 \%$ FBS. SW-626 and SK-OV-3 cells were cultured in Leibovitz's L15 medium and McCoy's 5a medium with 10\% FBS, respectively. Head and neck cancer cell lines were cultured in DMEM/ Ham's F12 50:50 mix medium with 10\% FBS. The cisplatin-resistant cells were purchased or generated by constant exposure of cells to cisplatin. KB-3-1 ${ }^{\text {cisR }}$, A549 ${ }^{\text {cisR }}$, and A2780 ${ }^{\text {cisR }}$ cells are 9.61-, 7.21-, and 7.76fold more resistant to cisplatin, respectively, than their parental cells in terms of cisplatin $\mathrm{IC}_{50}$ (41). HNSCC cell lines were a gift from G.Z. Chen and D.M. Shin (Emory University, Atlanta, Georgia, USA). The identity of HNSCC cell lines has been described previously (42-44). Lung cancer cell lines, ovarian cancer cell lines except A2780, and 293T cells were from the American Type Culture Collection. A2780 and A2780 ${ }^{\text {cisR }}$ cells were from Sigma-Aldrich. Cell line authentication was carried out using short tandem repeat profiling by RADIL CellCheck service.

Virus production, RNAi, and protein expression in human cancer cells. shRNA lentivirus was generated in 293T cells using pLKO.1 vector encoding shRNA, psPAX2, and pMD2.G. Cancer cells were infected with lentivirus for 2 days and selected using $2 \mu \mathrm{g} / \mathrm{ml}$ puromycin. Human ITPKB, NOX4, and IPMK were FLAG-tagged by PCR and subcloned into entry and destination vectors for expression. Hygromycin selection $(300 \mu \mathrm{g} / \mathrm{ml})$ was carried out for stable ITPKB expression. ITPKB shRNA-resistant silent mutant and kinase-dead mutant were generated by site-directed mutagenesis kit (Agilent Technologies).

RNAi-based screen. The primary screen was performed using the TRC Human Kinase shRNA Gene Family Library by infection of KB-3- $1^{\text {cisR }}$ cells with a lentivirus pool targeting individual gene and treating with $5 \mu \mathrm{g} / \mathrm{ml}$ of cisplatin (41). Among the 100 top ranking candidates from the screen, 16 kinases that use small metabolites as substrates were selected out and further investigated for synthetic lethality with cisplatin in A549 ${ }^{\text {cisR }}, \mathrm{A} 2780^{\text {cisR }}$, and $\mathrm{KB}-3-\mathrm{-}^{\mathrm{cisR}}$ cell lines.

Metabolic assays. Total intracellular ROS was determined by staining of the cells with $\mathrm{CM}-\mathrm{H}_{2}$ DCFDA. Intracellular $\mathrm{H}_{2} \mathrm{O}_{2}$ level (Promega), NADPH level (Promega), and GSH/GSSG ratio (EMD Millipore) were measured using commercial assay kits. Intracellular ATP levels were determined using ATP bioluminescent somatic cell assay (Sigma-Aldrich) and normalized to total cellular protein concentration. For RNA synthesis, subconfluent cells were spiked with $4 \mu \mathrm{Ci} / \mathrm{ml}$ of $\mathrm{D}$-[U-14 $\mathrm{C}]$ glucose for 2 hours. Total RNA was extracted, and the labeled RNA was quantified by liquid scintillation counting and normalized to the total RNA amount. For lipid synthesis, lipids were extracted using solvent of hexane and isopropanol (3:2 vol/vol). The solvent was air-dried and resuspended in chloroform. The amount of ${ }^{14} \mathrm{C}$-lipid was determined by liquid scintillation counting and normalized to the amount of protein. Glycolytic rate was determined by measurement of ${ }^{3} \mathrm{H}_{2} \mathrm{O}$ release. In brief, cells were spiked with $20 \mu \mathrm{Ci} / \mathrm{ml}$ of $\mathrm{D}^{-}\left[\mathrm{U}^{-3} \mathrm{H}\right]$ glucose in Krebs buffer supplemented with 10 $\mathrm{mM}$ glucose for 1 hour. Diffused ${ }^{3} \mathrm{H}_{2} \mathrm{O}$ from $\mathrm{D}-\left[\mathrm{U}-{ }^{3} \mathrm{H}\right]$ glucose was measured by liquid scintillation counting, and values were normalized with cell numbers of each sample. 
Enzyme activity assays. The activity of ITPKB was determined using ADP-Glo Kinase Assay (Promega) that measures ATP depletion. ITPKB variants were enriched from cell lysates and incubated with $10 \mu \mathrm{M}$ D-myo-IP3 in kinase assay buffer (50 mM Tris-HCl [pH 8], $100 \mathrm{mM}$ $\mathrm{NaCl}, 5 \mathrm{mM} \mathrm{MgCl}$, $1 \mathrm{mM}$ DTT, $10 \%$ glycerol, $10 \mu \mathrm{M} \mathrm{ATP,} 10 \mu \mathrm{M} \mathrm{CaCl}_{2}$ and $\mathrm{CaM}$ ) for 15 minutes, and luminescence was monitored. For cellular NOX activity assay, $100 \mu \mathrm{M}$ NADPH and $5 \mu \mathrm{M}$ lucigenin were added to $50 \mathrm{mg}$ of cellular membrane fraction for lucigenin chemiluminescence assay. In vitro NOX4 activity was measured by addition of $100 \mu \mathrm{M}$ NADPH to the reaction buffer ( $25 \mathrm{mM}$ HEPES [pH 7.3], $120 \mathrm{mM} \mathrm{NaCl}$, $3 \mathrm{mM} \mathrm{KCl}, 1 \mathrm{mM} \mathrm{MgCl}_{2}, 250 \mu \mathrm{M}$ flavin adenine dinucleotide) containing $30 \mu \mathrm{M}$ recombinant NOX4-DH and $10 \mu \mathrm{M}$ lucigenin as a final electron acceptor. Enzyme activities of GPX (BioVision), SOD (BioVision), TRX (Cayman), GSR (BioVision), and CAT (BioVision) were determined using commercially available kits according to the manufacturer's instructions.

Cell viability assay and colony formation assay. Cells were seeded at 5000 cells per well in 96-well plates 1 day before the addition of drugs with indicated concentrations for 48 hours. Cell viability was determined using CellTiter-Glo Luminescent Viability Assay (Promega). For colony formation assay, 250 cells were seeded in 35-mm dishes and treated with cisplatin for 24 hours. The cells were cultured in complete medium for another 10 days, and the colonies were stained with $0.5 \%$ crystal violet and counted by ImageJ software (NIH).

NMR spectroscopy. The intracellular concentrations of IP4 in $\mathrm{KB}-3-1^{\text {cisR }}$ cell lysates were measured by quantitative ${ }^{31} \mathrm{P}$ nuclear magnetic resonance (PNMR) spectroscopy (Bruker Avance III HD 600$\mathrm{MHz}$ spectrometer with a Prodigy Cryogen probe). $2 \times 10^{8}$ cells were dissolved in $0.5 \mathrm{ml}$ hypotonic lysis buffer $(0.5 \mathrm{mM}$ HEPES, $5 \mathrm{mM} \mathrm{KCl}$, $1 \mathrm{mM} \mathrm{MgCl}_{2}, 5 \mathrm{mM}$ DTT) and prepared using $\mathrm{D}_{2} \mathrm{O}$ with $0.74 \mathrm{M} \mathrm{H}_{3} \mathrm{PO}_{4}$ as an internal standard. The ${ }^{31} \mathrm{PNMR}$ spectra were acquired with ${ }^{1} \mathrm{H}$ decoupling and the delay time (d1) set to 10 seconds. Concentrations of metabolites were quantified based on the integrations of corresponding peaks versus $\mathrm{H}_{3} \mathrm{PO}_{4}$ peak and were normalized to the cell number.

Surface plasmon resonance. GST capture system and Biacore X100 (GE Healthcare) were used to perform surface plasmon resonance experiments. Anti-GST antibody was immobilized covalently to a CM5 sensor chip to capture the ligands, GST fused NOX4-DH or GST alone. Increasing concentrations of IP4 were prepared in 0.01 M HEPES ( $\mathrm{pH}$ 7.4), $0.005 \% \mathrm{vol} / \mathrm{vol}$ surfactant P20, $0.15 \mathrm{M} \mathrm{NaCl}$, and injected over GST or GST-NOX 4 at $30 \mu \mathrm{l} / \mathrm{min}$ for 180 seconds at $20^{\circ} \mathrm{C}$. Multiple-cycle kinetics analyses were used to quantify IP4 and GST-NOX4 interaction. The raw sensorgrams were obtained and subtracted with the values of GST alone. $K_{D}$ values were analyzed using BIA Evaluation Software.

Thermal shift assay. The NOX4 thermal shift experiment was performed using Protein Thermal Shift Dye Kit (Applied Biosystems). In brief, $30 \mu \mathrm{M}$ of purified recombinant NOX4-DH was incubated with increasing concentrations of IP4 or with $80 \mu \mathrm{M}$ of PIP3, IP3, IP4, and IP5 for 10 minutes at room temperature. The protein-metabolite mixture was applied to thermal shift reaction. The fluorescence data were collected on a real-time PCR system and analyzed using Protein Thermal Shift Software version 1.0.

Competitive binding assay. NADPH $(100 \mu \mathrm{M})$ and recombinant NOX4-DH $(30 \mu \mathrm{M})$ were incubated with IP4 in $1 \mathrm{ml}$ reaction buffer containing $25 \mathrm{mM}$ HEPES (pH 7.3), $120 \mathrm{mM} \mathrm{NaCl}, 3 \mathrm{mM} \mathrm{KCl}, 1$ $\mathrm{mM} \mathrm{MgCl}$, and $250 \mu \mathrm{M}$ flavin adenine dinucleotide for 30 minutes. NOX4-DH was eluted using FLAG M2 beads, and bound NADPH was quantified by measurement of absorbance at $340 \mathrm{~nm}$.
Immunohistochemistry staining. Formalin-fixed and paraffinembedded tissue from patients with HNSCC receiving platinum-based chemotherapy and tumors collected from xenograft mice were stained with anti-ITPKB (1:500) or anti-Ki-67 (1:1000) antibodies. ITPKB staining intensity and Ki-67 in the tumors were scored as 0 to 3.

Xenograft studies. Nude mice (Hsd:Athymic Nude-Foxn $1^{\text {nu }}$, female, 6 weeks old; Envigo) and NSG mice (NOD.Cg-Prkdcsid $I l 2 \mathrm{rg}^{\text {tmlWjil}} / \mathrm{SzJ}$, female, 6 weeks old; The Jackson Laboratory) were used for in vivo xenograft experiments. Nude mice were injected with $5 \times 10^{5} \mathrm{~KB}-3-1^{\text {cisR }}$ cells. Cisplatin $(5 \mathrm{mg} / \mathrm{kg})$ and GKT137831 $(30 \mathrm{mg} / \mathrm{kg})$ were administered twice a week by i.p. injection when tumor sizes reached up to $100-150 \mathrm{~mm}^{3}$. The NAC group was treated with $10 \mathrm{mg} / \mathrm{ml}$ NAC drinking water from 3 days after xenograft. The small cell lung carcinoma PDX and HNSCC PDX tumors were obtained from Emory University (45). Ovarian cancer PDX tumors were obtained from The Jackson Laboratory. The PDX tumors were implanted in the flank of 6-week-old nude mice or NOD scid gamma mice. The mice were randomly divided into groups when the tumor size reached $100-150 \mathrm{~mm}^{3}$. Cisplatin $(5 \mathrm{mg} / \mathrm{kg})$ and BAMB-4 (10 mg/kg) were given by i.p. injection twice a week. Tumors were measured blindly and the volume calculated as $4 \pi / 3 \times(\text { width } / 2)^{2} \times($ length $/ 2)$. Harvested tumors at the experimental endpoint were used for NOX activity and ROS detection. Tumor proliferation was assessed by Ki- 67 staining.

Assessment of nephrotoxicity. Biomarkers for kidney injury were examined every 3 days using commercial kits. Urine samples were collected and centrifuged at 9,000 $g$ for 5 minutes, and $5 \mu \mathrm{l}$ of supernatant was used for each assay. Fifty microliters of blood was collected to monitor serum creatine and blood urea nitrogen.

Statistics. Statistical parameters are indicated in the figure legends and figures. One representative experiment from multiple experiments is shown. Error bars represent mean \pm SEM for tumor volume curves and SD for all the rest. Statistical significance is based on 2-tailed Pearson correlation coefficient for Figure 1, B and C; 2-tailed Student's $t$ test for Supplemental Figure 6B, Supplemental Figure 7, A, B, and E, and Supplemental Figure 8, A (paired) and C; and 1-way or 2-way ANOVA with Bonferroni post hoc multiple-comparisons testing for all remaining data. $P$ values of 0.05 or less were considered statistically significant. Sample size was not predetermined using statistical methods. For in vivo study, animals were randomly chosen, and blinding outcome assessment and concealed allocation were used. The in vitro studies were not randomized, and allocation and outcome assessment were not blinded. Graphical presentation and statistical analyses were performed using GraphPad Prism 8.

Study approval. The present studies in human biological specimens were reviewed and approved by the Institutional Review Board (IRB00024810 and IRB00003208) at Emory University, Atlanta, Georgia, USA. The study was conducted in compliance with all ethical standards and good clinical practice. Tissue specimens for the immunohistochemistry study were obtained from the Head and Neck Satellite Tissue Bank at Emory University. The bank collects tissue specimens from adult patients aged over 21 who have HNSCC as identified by the Emory Oral Pathology Biopsy Service. All clinical samples were collected with written informed consent of the participants or their guardians. In detail, tumors were surgically resected at Emory University Hospital, formalin-fixed, paraffinembedded, and archived. Clinical information on the specimens was obtained from the surgical pathology files following the regula- 
tions of HIPAA. Animal experiments were performed according to a protocol approved by the Institutional Animal Care and Use Committee of Emory University.

\section{Author contributions}

GZC, NFS, DMS, TKO, and SL provided critical reagents. LZ performed structural analyses. KRM and XW performed histopathological study and provided clinical information. YL and HM carried out NMR experiments. GZ performed patient-derived xenograft. HBK generated virus for RNAi screen. CP, LJ, JC, ACB, and DL performed all the other experiments. CP and SK designed the study and wrote the paper.

\section{Acknowledgments}

We acknowledge Anthea Hammond for editorial assistance. This work was supported in part by NIH grants R01 CA175316 (to SK) and R01 CA207768 (to SK), Department of Defense grant
W81XWH-17-1-0186 (to SK), Developmental Funds from the Winship Cancer Institute of Emory University (to SK), Winship Cancer Institute IRG-17-181-04 from the American Cancer Society (to LJ), and the Emory University Integrated Cellular Imaging Microscopy Core of the Winship Cancer Institute Comprehensive Cancer Center, grant 2P30CA138292. SK is a Georgia Cancer Coalition Scholar, a Robbins Scholar, and an American Cancer Society Basic Research Scholar.

Address correspondence to: Sumin Kang, Emory University School of Medicine, 1365-C Clifton Road NE, Atlanta, Georgia 30322, USA. Phone: 404.778.1880; Email: smkang@emory.edu.

LJ's present address is: Department of Anatomy and Cell Biology, University of Florida, Gainesville, Florida, USA.

LZ's present address is: School of Pharmacy, Fudan University, Shanghai, China.
1. Barabas K, Milner R, Lurie D, Adin C. Cisplatin: a review of toxicities and therapeutic applications. Vet Comp Oncol. 2008;6(1):1-18.

2. Kelland L. The resurgence of platinum-based cancer chemotherapy. Nat Rev Cancer. 2007;7(8):573-584.

3. Rosenberg B, VanCamp L, Trosko JE, Mansour VH. Platinum compounds: a new class of potent antitumour agents. Nature. 1969;222(5191):385-386.

4. Liu H, Liu Y, Zhang JT. A new mechanism of drug resistance in breast cancer cells: fatty acid synthase overexpression-mediated palmitate overproduction. Mol Cancer Ther. 2008;7(2):263-270.

5. Zhou M, et al. Warburg effect in chemosensitivity: targeting lactate dehydrogenase-A re-sensitizes taxol-resistant cancer cells to taxol. $\mathrm{Mol}$ Cancer. 2010;9:33.

6. Montopoli M, et al. "Metabolic reprogramming" in ovarian cancer cells resistant to cisplatin. Curr Cancer Drug Targets. 2011;11(2):226-235.

7. Shirmanova MV, et al. Chemotherapy with cisplatin: insights into intracellular $\mathrm{pH}$ and metabolic landscape of cancer cells in vitro and in vivo. Sci Rep. 2017;7(1):8911.

8. Lee MY, et al. Reprogrammed lipid metabolism in bladder cancer with cisplatin resistance. Oncotarget. 2018;9(17):13231-13243.

9. Szatrowski TP, Nathan CF. Production of large amounts of hydrogen peroxide by human tumor cells. Cancer Res. 1991;51(3):794-798.

10. Toyokuni S, Okamoto K, Yodoi J, Hiai H. Persistent oxidative stress in cancer. FEBS Lett. 1995;358(1):1-3.

11. Trachootham D, Alexandre J, Huang P. Targeting cancer cells by ROS-mediated mechanisms: a radical therapeutic approach? Nat Rev Drug Discov. 2009;8(7):579-591.

12. Ai Z, Lu Y, Qiu S, Fan Z. Overcoming cisplatin resistance of ovarian cancer cells by targeting HIF-1-regulated cancer metabolism. Cancer Lett. 2016;373(1):36-44.

13. Rytelewski M, et al. BRCA2 inhibition enhances cisplatin-mediated alterations in tumor cell proliferation, metabolism, and metastasis. $\mathrm{Mol}$ Oncol. 2014;8(8):1429-1440.

14. Liang XJ, Finkel T, Shen DW, Yin JJ, Aszalos A,
Gottesman MM. SIRT1 contributes in part to cisplatin resistance in cancer cells by altering mitochondrial metabolism. Mol Cancer Res. 2008;6(9):1499-1506.

15. Wangpaichitr M, et al. The relationship of thioredoxin-1 and cisplatin resistance: its impact on ROS and oxidative metabolism in lung cancer cells. Mol Cancer Ther. 2012;11(3):604-615.

16. Itoh $\mathrm{T}$, et al. Cisplatin induces production of reactive oxygen species via NADPH oxidase activation in human prostate cancer cells. Free Radic Res. 2011;45(9):1033-1039.

17. Shono T, et al. Enhanced expression of NADPH oxidase Nox4 in human gliomas and its roles in cell proliferation and survival. Int J Cancer. 2008;123(4):787-792.

18. Kim HJ, et al. Roles of NADPH oxidases in cisplatin-induced reactive oxygen species generation and ototoxicity. JNeurosci. 2010;30(11):3933-3946.

19. Meng XM, et al. NADPH oxidase 4 promotes cisplatin-induced acute kidney injury via ROS-mediated programmed cell death and inflammation. Lab Invest. 2018;98(1):63-78.

20. Chamberlain PP, Sandberg ML, Sauer K, Cooke MP, Lesley SA, Spraggon G. Structural insights into enzyme regulation for inositol 1,4,5-trisphosphate 3-kinase B. Biochemistry. 2005;44(44):14486-14493.

21. Irvine RF, Schell MJ. Back in the water: the return of the inositol phosphates. Nat Rev Mol Cell Biol. 2001;2(5):327-338.

22. Shears SB. How versatile are inositol phosphate kinases? Biochem J. 2004;377(pt 2):265-280.

23. Schröder D, et al. The new InsP3Kinase inhibitor BIP-4 is competitive to InsP3 and blocks proliferation and adhesion of lung cancer cells. Biochem Pharmacol. 2015;96(2):143-150.

24. Schröder D, Rehbach C, Seyffarth C, Neuenschwander M, Kries JV, Windhorst S. Identification of a new membrane-permeable inhibitor against inositol-1,4,5-trisphosphate-3-kinase A. Biochem Biophys Res Commun. 2013;439(2):228-234.

25. Miller AT, et al. Inhibition of the inositol kinase Itpkb augments calcium signaling in lymphocytes and reveals a novel strategy to treat autoimmune disease. PLoS One. 2015;10(6):e0131071.
26. Maréchal Y, et al. Inositol 1,3,4,5-tetrakisphosphate controls proapoptotic Bim gene expression and survival in B cells. Proc Natl Acad Sci U S A. 2007;104(35):13978-13983.

27. Maréchal Y, Quéant S, Polizzi S, Pouillon V, Schurmans S. Inositol 1,4,5-trisphosphate 3-kinase B controls survival and prevents anergy in B cells. Immunobiology. 2011;216(1-2):103-109.

28. Miller AT, et al. Production of Ins $(1,3,4,5)$ P4 mediated by the kinase Itpkb inhibits store-operated calcium channels and regulates B cell selection and activation. Nat Immunol. 2007;8(5):514-521.

29. Wen BG, et al. Inositol $(1,4,5)$ trisphosphate 3 kinase B controls positive selection of T cells and modulates Erk activity. Proc Natl Acad Sci U S A. 2004;101(15):5604-5609.

30. Siegemund S, et al. IP3 3-kinase B controls hematopoietic stem cell homeostasis and prevents lethal hematopoietic failure in mice. Blood. 2015;125(18):2786-2797.

31. Windhorst S, et al. Inositol 1,4,5-trisphosphate 3-kinase-A is a new cell motility-promoting protein that increases the metastatic potential of tumor cells by two functional activities. J Biol Chem. 2010;285(8):5541-5554.

32. Edlich F, et al. Bcl-x(L) retrotranslocates Bax from the mitochondria into the cytosol. Cell. 2011;145(1):104-116.

33. Meshkini A, Yazdanparast R. Involvement of oxidative stress in taxol-induced apoptosis in chronic myelogenous leukemia K562 cells. Exp Toxicol Pathol. 2012;64(4):357-365.

34. Jin L, et al. Glutamate dehydrogenase 1 signals through antioxidant glutathione peroxidase 1 to regulate redox homeostasis and tumor growth. Cancer Cell. 2015;27(2):257-270.

35. Jin L, et al. The PLAG1-GDH1 axis promotes anoikis resistance and tumor metastasis through CamKK2-AMPK signaling in LKB1-deficient lung cancer. Mol Cell. 2018;69(1):87-99.e7.

36. Boonstra J, Post JA. Molecular events associated with reactive oxygen species and cell cycle progression in mammalian cells. Gene. 2004;337:1-13.

37. Schieber M, Chandel NS. ROS function in redox signaling and oxidative stress. Curr Biol. 2014;24(10):R453-R462. 
38. Block K, Gorin Y. Aiding and abetting roles of NOX oxidases in cellular transformation. Nat Rev Cancer. 2012;12(9):627-637.

39. Kawai Y, Nakao T, Kunimura N, Kohda Y, Gemba M. Relationship of intracellular calcium and oxygen radicals to Cisplatin-related renal cell injury. JPharmacol Sci. 2006;100(1):65-72.

40. Chang G, Chen L, Lin HM, Lin Y, Maranchie JK. Nox4 inhibition enhances the cytotoxicity of cisplatin in human renal cancer cells. J Exp Ther
Oncol. 2012;10(1):9-18.

41. Jin $\mathrm{L}$, et al. MAST1 drives cisplatin resistance in human cancers by rewiring cRaf-independent MEK activation. Cancer Cell. 2018;34(2):315-330.e7.

42. Kang S, et al. p90 ribosomal S6 kinase 2 promotes invasion and metastasis of human head and neck squamous cell carcinoma cells. JClin Invest. 2010;120(4):1165-1177.

43. Zhao M, et al. Assembly and initial characterization of a panel of 85 genomically validated cell lines from diverse head and neck tumor sites. Clin Cancer Res. 2011;17(23):7248-7264.

44. Lin CJ, et al. Head and neck squamous cell carcinoma cell lines: established models and rationale for selection. Head Neck. 2007;29(2):163-188.

45. Owonikoko TK, et al. Patient-derived xenografts faithfully replicated clinical outcome in a phase II co-clinical trial of arsenic trioxide in relapsed small cell lung cancer. J Transl Med. 2016;14(1):111. 